\title{
Response predictions using the observed autocorrelation function
}

\author{
Nielsen, Ulrik Dam; H. Brodtkorb, Astrid; Jensen, Jørgen Juncher
}

Published in:

Marine Structures

Link to article, DOI:

10.1016/j.marstruc.2017.10.012

Publication date:

2018

Document Version

Peer reviewed version

Link back to DTU Orbit

Citation (APA):

Nielsen, U. D., H. Brodtkorb, A., \& Jensen, J. J. (2018). Response predictions using the observed autocorrelation function. Marine Structures, 58, 31-52. https://doi.org/10.1016/j.marstruc.2017.10.012

\section{General rights}

Copyright and moral rights for the publications made accessible in the public portal are retained by the authors and/or other copyright owners and it is a condition of accessing publications that users recognise and abide by the legal requirements associated with these rights.

- Users may download and print one copy of any publication from the public portal for the purpose of private study or research.

- You may not further distribute the material or use it for any profit-making activity or commercial gain

- You may freely distribute the URL identifying the publication in the public portal 


\title{
Response predictions using the observed autocorrelation function
}

\author{
Ulrik D. Nielsen ${ }^{\mathrm{a}, \mathrm{b}}$, Astrid H. Brodtkorb ${ }^{\mathrm{b}}$, Jørgen J. Jensen ${ }^{\mathrm{a}}$ \\ ${ }^{a}$ DTU Mechanical Engineering, Technical University of Denmark, DK-2800 Kgs. Lyngby, \\ Denmark \\ ${ }^{b}$ Centre for Autonomous Marine Operations and Systems, AMOS-NTNU, NO-7491 \\ Trondheim, Norway
}

\begin{abstract}
This article studies a procedure that facilitates short-time, deterministic predictions of the wave-induced motion of a marine vessel, where it is understood that the future motion of the vessel is calculated ahead of time. Such predictions are valuable to assist in the execution of many marine operations (crane lifts, helicopter landings, etc.), as a specific prediction can be used to inform whether it is safe, or not, to carry out the particular operation within the nearest time horizon. The examined prediction procedure relies on observations of the correlation structure of the wave-induced response in study. Thus, predicted (future) values ahead of time for a given time history recording are computed through a mathematical combination of the sample autocorrelation function and previous measurements recorded just prior to the moment of action. Importantly, the procedure does not need input about the exciting wave system, and neither does it rely on off-line training. In the article, the prediction procedure is applied to experimental data obtained through model-scale tests, and the procedure's predictive performance is investigated for various irregular wave scenarios. The presented results show that predictions can be successfully made in a time horizon corresponding to about 8-9 wave periods ahead of current time (the moment of action).

Keywords:
\end{abstract}

Email address: udn@mek.dtu.dk (Ulrik D. Nielsen)

Preprint submitted to Marine Structures

July 10, 2017 
Determinstic motion prediction, real-time, measurements, stationary process, sample autocorrelation function, conditional process

\section{Introduction}

2

Most marine operations require a high level of safety. This is also the case when concern is for here-and-now operations such as lifts by floating cranes, helicopter landings on (smaller) ships, tow of drilling and production vessels/platforms, and various ship-to-ship actions. The execution of these operations can be made safer if the particular vessels wave-induced motions can be predicted ahead of current time. Thus, the ability to calculate accurately, in a deterministic sense, the future wave-induced behaviour of the vessel can reduce significantly the probability of failure of the actual operation. Some of the before mentioned operations involve dynamically positioned (DP) vessels and one means to apply the predicted response/motion ahead of current time can, in this case, be used directly in proactive control strategies for the DP system. Examples of strategies may be to adjust the controller gains, change the set-point of smaller vessels, and for larger vessels accelerate the vessel into the waves to avoid drift-off, or, if worst comes to worst, have sufficient time to emergency-abort the operation safely. Other practices, where the prediction of vessel motions ahead of current time is valuable, occur for general heave compensation systems, and for robotic manipulators on ships and other seaborne platforms, since efficient operation of the manipulators requires precise motion planing and control algorithms. As a practical remark, it should be noted that current time, in the following, relates to the very instant from when a prediction is made, meaning that measurements have been recorded (are known) only until the current time. Equivalently, this specific time could be defined as the moment of action from when the future (hydrodynamic) behaviour of the vessel is predicted.

\subsection{Previous work}

In the past, a number of studies has been conducted to investigate procedures for the prediction of the wave-induced motion of a marine vessel. Some of 
the initial studies, e.g., Dalzell [1], Triantafyllou et al. [2, 3] and Sidar and Doolin [4], were concerned about the landing of aircrafts on naval destroyers. Since then, several of other works have followed both with naval and merchant applications; for instance, Broome [5], Broome and Hall [6], Chung et al. [7], Duan et al. [8], From et al. [9], Khan et al. [10, 11, 12], Naaijen et al. [13], Peng et al. [14], Woodacre et al. [15], Zhao et al. [16]. Most works in the existing literature belong to one of two main categories. Either the established prediction procedure relies on; a) a combined knowledge of the exciting wave system and the hydrodynamic behaviour of the ship, e.g. in terms of the ships transfer function, or b) the procedure relies on some sort of offline training which is necessary for 'standard' autoregressive (AR) models and Neural Networks that, on the other hand, not necessarily require input about the waves/sea state. Obviously, independence of (information about) the sea state is beneficial, as real-time ocean surface and sea state estimation, at a ship's exact location, in itself can be a difficult problem to handle in practice, not to mention the uncertainty associated to the actual estimate produced by whatever estimating means $[17,18,19]$.

It is possible to formulate a prediction procedure, see Andersen et al. [20], which neither requires information about the wave conditions, nor does it require offline training. In the particular procedure - for any considered motion component - the sample autocorrelation function (ACF) for a recent time window needs to be obtained. The (sampled) ACF must represent a stationary situation which, in time and properties, is so close to the current time that the statistics and the correlation structure in the dynamical system have not changed significantly. Thus, leaving the basic details for later, the prediction procedure relies on a linear model based on the correlation structure, in terms of the autocorrelation function, of the physical process in question together with the most recent - past - measurement points. In this connection, it is important to realise that the autocorrelation function is a direct measure of the physical process' underlying memory effect; here due to the free surface oscillations of the sea surface. Another property to keep in mind, when discussing a process's 
memory and the autocorrelation function, is the fact that, for a stationary process, "... the autocorrelation function and the spectrum are transforms of each other, (hence) they are mathematically equivalent" [21]. This fact is made directly use of later, but, as a qualitative interpretation of the property, it means that an infinitely narrow-banded process has infinitely long prediction horizon; since the process has, in the extreme case, one single frequency component and, hence, is described by a sine wave. The opposite is true for an infinitely broadbanded process (i.e. white noise), where the deterministic prediction horizon is zero.

In a recent study, Nielsen and Jensen [22] investigated the procedure, [20], to predict vessel responses up to 50 seconds ahead of current time. The study [22] was focused on simulated time histories of a ship's wave-induced vertical acceleration at the centreline at a longitudinal position forward of the COG. In total, $20 \times 60$ minutes of measurements data were simulated, and predictions, looking 50 seconds ahead, were made every 10 seconds within the single 60minutes time strips. Hence, 7,200 $(=3,600 \mathrm{~s} / 10 \mathrm{~s} \times 20)$ sets of \{predictions vs. measurements $\}$ were analysed and statistically evaluated. The study showed that predictions of the acceleration level could be successfully made up to 20 seconds ahead of time for most of the sets (about 85-90\%); however, with prediction accuracy reducing beyond this time to a success rate of $10-20 \%$ at the end of the prediction intervals (spanning 50 seconds). Various metrics were derived to establish the statistical comparison between the predictions and the (simulated) measurements but, obviously, there is no unique way of doing the comparison of individual time history strips; a fact which also will be addressed later in the present study.

\subsection{Content of the study}

The investigated procedure by Nielsen and Jensen [22] is also examined in the present study but, herein, the measurements data consist of motion recordings obtained from model-scale experiments rather than numerically simulated time histories. Some of the findings made in [22] are directly applied in the present 
work and, as as such, the study herein is a continuation of the former one, including the recommended further work.

In most studies on stochastic wave-structure interactions, the statistical concept of a stationary process is important. Indeed, this is so herein and throughout it is a fundamental assumption that conditions are stationary. In principle, this calls for a discussion on requirements for a process to be stationary, or maybe rather a discussion of the theoretical/mathematical consequences if the process is not strictly (nor weakly) stationary. However, this particular discussion is not touched upon, although some remarks are given. Overall, the importance is that stationarity will be assumed; without necessarily stating this.

It should also be mentioned that the interest in this study concerns 'standard marine crafts', such as ships or other ship-like structures and floating platforms, and not tethered marine structures. On the other hand, the theoretical formulations might apply to the latter type of structures; if the particular response is characterised by a (Gaussian) stationary process.

\subsection{Composition of paper}

The paper has been organised into five main sections, and the remaining four are as follows: In Section 2, the theoretical formulations are outlined with mentioning also about general properties about the (sample) autocorrelation function of a stationary process. The experimental facility, including descriptions of the test cases, and pre-analyses of the recorded model-scale data are described in Section 3. All predictions, and associated results and comparisons with measurements, follow in Section 4. Finally, a short summary and an extraction of main findings and conclusions are given in Section 5.

\section{Theoretical formulation}

The prediction procedure addressed herein is established from the fact that any stationary wave-induced response has some memory in its behaviour. The 
reminiscence arises due to a memory effect in the exciting force which is governed by the wave oscillations of the sea surface. The ability of a wave-induced process to "remember its past" may conversely be expressed by saying that the future values (= outcomes) of the particular process will be conditional on its prior outcomes. Thus, it makes sense to introduce the (statistical) concept of conditional processes, and the prediction procedure makes directly use of results which can be derived from the definition of the joint probability density function of time-dependent normal distributed variables. Indeed, Lindgren [23] studied properties of a normal process, and results and general findings were outlined for the conditional behaviour of a normal process. The mentioned study [23] is at a somewhat high-level of mathematical abstraction/notation, and some of the findings have been concretised by Jensen [24] and Andersen et al. [20]. The following section outlines the expressions that have been derived for a normal process, conditional on a set of known, i.e., (prior) measured values.

The main focus in the article is on application; rather than going through all the details of the theory. That said, all relevant and necessary theory is included in the following, but some of the algebra, derived from the original work by Lindgren [23], has been left out. In order to assist the reader, the relevant theory has been compressed down to a set of bullet points specified in subsection 2.2 , and the reader may jump directly to this subsection.

\subsection{Conditional process based on a set of known values}

The measurement $x\left(t_{0}\right)=x_{0}$ of an arbitrary wave-induced response at an instant $t_{0}$ is considered, and measured values $x\left(t_{1}\right)=x_{1}, x\left(t_{2}\right)=x_{2}, \ldots, x\left(t_{n}\right)=$ $x_{n}$ exist at a set of times $t_{1}>t_{2}>\ldots>t_{n}$ prior to $t_{0}$. Mathematically, the measurements are described by the stochastic normal process $X(t)$, and the interest is concerned with the expected mean variation $\widehat{X(t)}$ of $X(t)$ ahead of current time (i.e. $t>t_{0}$ ). By definition, the expected mean variation of the 
conditional process, conditioning $X(t)$ on its prior values, is given by

$$
\begin{aligned}
\widehat{X(t)} & \equiv E\left[X(t) \mid X\left(t_{0}\right)=x_{0}, X\left(t_{1}\right)=x_{1}, \ldots, X\left(t_{n}\right)=x_{n}\right] \\
& =\int_{-\infty}^{\infty} u \cdot p\left(u \mid x_{0}, x_{1}, \ldots, x_{n}\right) d x
\end{aligned}
$$

where $p\left(x \mid x_{0}, x_{1}, \ldots, x_{n}\right)$ is the conditional probability density function of $X(t)$ given $X\left(t_{0}\right)=x_{0}, X\left(t_{1}\right)=x_{1}, \ldots, X\left(t_{n}\right)=x_{n}$. Since the probability density function of $X(t)$ is normal distributed, the conditional probability density function will also be (multivariate) normal distributed,

$$
p\left(x \mid x_{0}, x_{1}, \ldots, x_{n}\right)=\varphi\left(x(t) ; \mu_{n}(t), \sigma_{n}(t)\right)
$$

where $\varphi(x ; \mu, \sigma)$ is the probability density function of a normal distributed variable

$$
\varphi(x ; \mu, \sigma) \equiv \frac{1}{\sqrt{2 \pi} \sigma} \exp \left(-\frac{1}{2}\left(\frac{x-\mu}{\sigma}\right)^{2}\right)
$$

with mean value $\mu$ and standard deviation $\sigma$. In the particular case, Eq. (2), the mean value and the standard deviation are themselves processes rather than variables. Notably, the 'mean value' will be identical to the expected mean variation, i.e. $\mu_{n}(t) \equiv \widehat{X(t)}$, which is the very solution to the prediction problem. The derivation of the explicit formula for $\widehat{X(t)}$, generally expressed in terms of $n$ prior measured values of $X(t)$, requires some algebra. Below, the solution will be indicated only for the special case $n=1$.

The conditional probability density function of the process $X(t)$, given $X\left(t_{0}\right)=$ $x_{0}$ and $X\left(t_{1}\right)=x_{1}$, can be written, cf. Jensen [24]

$$
p\left(x(t) \mid x_{0}, x_{1}\right)=\frac{p\left(x(t), x_{0}, x_{1}\right)}{p\left(x_{0}, x_{1}\right)}
$$

Thus, the interest is in the marginal probability density functions, $p\left(x_{0}, x_{1}\right)$ and $p\left(x(t), x_{0}, x_{1}\right)$, which both are multivariate versions of the normal distribution. For the $k$-variate case, with $\mathbf{x}$ being a vector of $k$ elements, the expression reads

$$
p(\mathbf{x})=\frac{1}{\sqrt{|(2 \pi) \boldsymbol{\Sigma}|}} \exp \left(-\frac{1}{2}\left(\mathbf{x}^{T} \boldsymbol{\Sigma}^{-1} \mathbf{x}\right)\right)
$$

where $\boldsymbol{\Sigma}$ is the (auto)covariance matrix of $p(\mathbf{x})$, and $|\cdot|$ denotes determinant. In Eqs. (4)-(5), the autocovariance matrices $\boldsymbol{\Sigma}_{2}$ and $\boldsymbol{\Sigma}_{3}$ for $p\left(x_{0}, x_{1}\right)$ and 
165 $168 r(t)$,

169

170

where $r(t)$ is given by,

$$
r(t)=\frac{1}{m_{0}} E[X(0) X(t)]
$$

171

introducing the variance in terms of the 0 -th order spectral moment $m_{0}$, and noting $r\left(t_{1}-t\right)=r\left(t-t_{1}\right)$ for a stationary process. The i-th order spectral moment $m_{i}$ follows from

$$
m_{i}=\int_{0}^{\infty} \omega^{i} S(\omega) d \omega
$$

with the spectral density $S(\omega)$, at frequency $\omega$, being the Fourier transform of the (stationary) time domain process $X(t)$. It is noteworthy that the definition of the time-dependent autocorrelation function (Eq. 10) is formulated in the time domain, but for a stationary process the autocorrelation function may alternatively be obtained by a frequency domain calculation,

$$
r(t)=\frac{1}{m_{0}} \int_{0}^{\infty} S(\omega) \cos (\omega t) d \omega
$$

(1) 1

The further steps in the development of the prediction procedure are to insert Eq. (8) and Eq. (9), respectively, into Eq. (5), yielding analytical expressions for the two marginal probability density functions $p\left(x_{0}, x_{1}\right)$ and 
$p\left(x(t), x_{0}, x_{1}\right)$. Subsequently, substitution of these two expressions into Eq. (4) leads - through (algebraic) matrix multiplication - to an analytic expression for the conditional probability density function $p\left(x(t) \mid x_{0}, x_{1}\right)$. On the other hand, the assumption is that $p\left(x(t) \mid x_{0}, x_{1}\right)$ is given by a normal probability density function, $\varphi\left(x(t) ; \mu_{1}(t), \sigma_{1}(t)\right)$, with given processes for the mean value $\mu_{1}(t)$ and the standard deviation $\sigma_{1}(t)$, cf. Eqs. (2) and (3). Hence, from the (explicit) analytic expression of the conditional probability density function it is possible to define analytic expressions for $\mu_{1}(t)$ and $\sigma_{1}(t)$; keeping in mind that the former yields the actual prediction in search, $\widehat{X(t)}=\mu_{1}(t)$. Thus, the expected mean variation, equivalently said the prediction ahead of current time $t_{0}$, can be calculated from

$$
\begin{aligned}
\widehat{X(t)} & =\frac{\left(r(t)-r\left(t_{1}\right) r\left(t-t_{1}\right)\right) x_{0}+\left(r\left(t-t_{1}\right)-r(t) r\left(t_{1}\right)\right) x_{1}}{1-r^{2}\left(t_{1}\right)} \\
& =\frac{1}{1-r^{2}\left(t_{1}\right)}\left[r(t), r\left(t-t_{1}\right)\right]\left[\begin{array}{cc}
1 & -r\left(t_{1}\right) \\
-r\left(t_{1}\right) & 1
\end{array}\right]\left[x_{0}, x_{1}\right]^{T}
\end{aligned}
$$

In the formula above, only the two most recent measurements, $x_{0}$ and $x_{1}$, are taken into account. In the general case with a set of $n$ prior values, that is $n>1$, the formula for predictions ahead of time $t_{0}$ changes accordingly:

$$
\widehat{X(t)}=\mathbf{r}^{T}(t) \mathbf{R}^{-1} \mathbf{x}
$$

using matrix notation with the 'measurement vector' $\mathbf{x}=\left[x_{0}, x_{1}, x_{2}, \ldots, x_{n}\right]^{T}$. For at discrete set of (lagged) times, $t_{k}=k \Delta t, k=0,1,2, \ldots, n$ (i.e. $t_{0}=0$ ), the autocorrelation vector $\mathbf{r}(t)$ and autocorrelation matrix $\mathbf{R}$ are,

$$
\mathbf{r}(t)=[r(t-0), r(t-\Delta t), r(t-2 \Delta t), \ldots, r(t-n \Delta t)]^{T}
$$

$$
\mathbf{R}=\left[\begin{array}{ccccc}
1 & r(\Delta t) & r(2 \Delta t) & \cdots & r(n \Delta t) \\
r(\Delta t) & 1 & r(\Delta t) & \cdots & r((n-1) \Delta t) \\
r(2 \Delta t) & r(\Delta t) & 1 & \cdots & r((n-2) \Delta t) \\
\vdots & \vdots & \vdots & \ddots & \vdots \\
r(n \Delta t) & r((n-1) \Delta t) & \cdots & r(\Delta t) & 1
\end{array}\right]
$$


where it is noted that the autocorrelation matrix is symmetric, with constant elements on any diagonal, and with ones on the centre diagonal. The autocorrelation (row) vector has length $n+1$, while the autocorrelation (square) matrix has dimension $(n+1) \times(n+1)$. For $n=1$ it is evident that Eq. (14) becomes identical to Eq. (13).

\subsection{Summary}

The prediction procedure is complete with expression Eq. (14). As a kind of summary, and with attention to calculations in practice, where stationary conditions will be assumed, a few important points are worth mentioning:

1. The autocorrelation matrix (Eq. 16) does not change, and it needs therefore to be calculated and inverted only once for the considered range of stationary data.

2. The autocorrelation vector (Eq. 15) does not depend on the (instantaneous) measured values of $X(t)$ and can be precalculated and re-used for the set of prior time steps considered at the particular time step(s) of the (discretised) time $t$.

3. Combining (1) and (2) leads to the 'predictive vector' $\mathbf{y}(t)$ which is precalculated, or adapted, to the particular setup of prediction horizon and prior measurements considered,

$$
\mathbf{y}(t)=\mathbf{r}^{T}(t) \mathbf{R}^{-1}, \operatorname{size}(\mathbf{y})=1 \times(n+1) .
$$

4. In practice, one specific vector, $\mathbf{y}_{m}, m=1,2, \ldots, M$, is computed/assigned corresponding to one particular time $t_{m}$ ahead of current time $t_{0}$. Thus, on a discrete time interval, $t_{m}, m=1,2, \ldots, M$, predictions of the process $X(t)$ are calculated according to

$$
\widehat{x}_{m}=\mathbf{y}_{m} \mathbf{x}
$$

noting that $\mathbf{y}_{m}=\left[y_{1, m}, y_{2, m}, \ldots, y_{n+1, m}\right]$ and $\mathbf{x}=\left[x_{0}, x_{1}, x_{2}, \ldots, x_{n}\right]^{T}$. 
The outlined prediction procedure has some resemblance to predictions by the autoregressive (AR) predictor method, e.g. Zhao et al. [16], but with a main difference that for the AR procedure the future time step is assumed to be only the next time step and not a continuous variable as in Eq. (14) (and Eq. 18). Consequently, any 'standard' AR procedure needs some sort of offline training to facilitate predictions at several time steps ahead of current time. In contrast, the present prediction procedure (Eq. 14) makes directly use of the system's correlation structure in terms of the autocorrelation function and, thus, offline training is not needed to make predictions at a number of time steps ahead of time.

Some additional discussions, including comparisons, of theoretical concepts of various prediction procedures are given in, for instance, [20] and [9]. The present section is closed by a small theoretical example/illustration which serves to explain some general aspects of calculations, i.e. predictions, made with Eq. (14).

\subsection{Theoretical example}

A certain response has been monitored and recorded during a stationary period. Specifically, a time history recording of the past 30 minutes (see Figure 1) has been used to estimate the response spectrum and the associated autocorrelation function. Some three minutes later is considered as the current time, i.e. "now", where a prediction ahead of time is made. Figure 1 shows the situation; the upper plot is the 30-minutes time recording while the lower plot is a zoom around the current time, which is taken to be three minutes later than the end of the 30-minutes time history recording providing the underlying correlation structure.

At time $t_{0}=33.0$ min., a value $x_{0}$ is measured and predictions ahead of $t_{0}$ are made using the past, say, $T_{\text {past }}=20$ seconds of data. Thus, with the sampling rate to be, for instance, $\Delta t=0.5 \mathrm{~s}$, it means that 41 prior points are considered for predictions, and the measurement vector $\mathbf{x}_{33 \min }=$ $\left[x_{0}, x_{1}, \ldots, x_{40}\right]^{T}$ is assigned accordingly. The autocorrelation matrix $\mathbf{R}_{20 s}$ has 

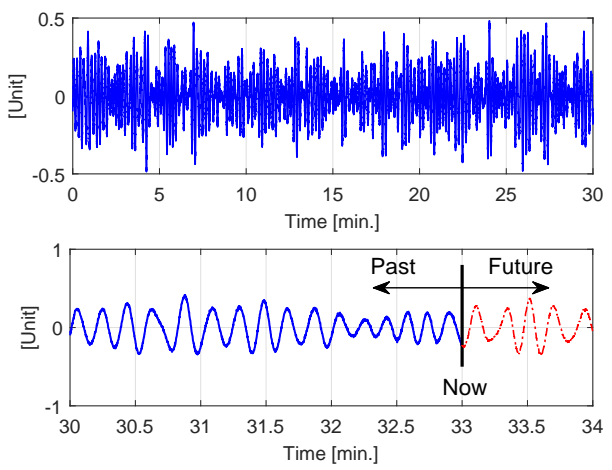

Figure 1: Response measurements recorded during 30 minutes of stationary conditions (upper plot) and a zoom around current time $t_{0}=33.0 \mathrm{~min}$. at which a prediction of the future behaviour is made.

been (pre)constructed with its $41 \times 41$ elements, cf. Eq. (16), using the calculated autocorrelation function derived from the 30-minutes (stationary) time recording. At any one time step $(m \Delta t), m=1,2, \ldots$ ahead of $t_{0}$ the predictive vector $\mathbf{y}_{20 s, m}=\left[y_{1}, y_{2}, \ldots, y_{41}\right]_{m}$ has 41 elements and it is calculated according to Eq. (17), noting that the vector depends only on the autocorrelation function, depending itself on just the initial 30-minutes time history recording. In this scenario, however, prediction is made $T_{\text {predict }}=60$ seconds ahead of $t_{0}$, so a set of $120\left(=\frac{T_{\text {predict }}}{\Delta t}\right)$ predictive vectors is needed; a set that can be stored as a matrix $\mathbf{Y}_{20 s, 60 s}$, which will be specific to the combination of $T_{\text {past }}$ and $T_{\text {predict }}$.

As a consequence of the above "deduction", any new predictions, made also 60 seconds ahead of a 'new current time' being different from $t_{0}$, can be made by just changing $\mathbf{x}$, since $\mathbf{y}$ has not changed; assuming no change in the correlation structure of the process at the new current time. More generally, from the illustration-example, it is important to note (and to repeat) that in the prediction procedure;

- the measurement vector $\left(\mathbf{x}_{t_{0}}\right)$ will be specific to the instant in time when predictions are made,

- the autocorrelation function is specific only to the considered stationary 
time history recording; and thus the (specific) autocorrelation matrix $\mathbf{R}$, independent of the value of $t_{0}$, can be used for predictions as long as conditions remain in the same 'stationary settings',

- consequently, or similarly, any predictive vector $\mathbf{y}$ does not change with the current time $t_{0}$, whatever the value of $t_{0}$, requiring just that $t_{0}$ is not (very) far away, measured on a time scale, from the initial stationary time history recording.

As a closing remark on the theoretical example, but focusing instead on the practical application of a predicted response sequence, one means to exploit such deterministic predictions (e.g., Fig. 1) is to provide the maximum and minimum values of the predicted time sequence. That is, it may not necessarily be important to know that, say, the heave motion will be $+0.98 \mathrm{~m}, 28$ seconds ahead of current time. Rather it will be beneficial to know that it is likely that the heave motion, during the next, say, 30 seconds, reaches a specific level (plus/minus) that makes a particular operation unsafe to carry out. Obviously, for a perfect prediction procedure the term 'likely' will be replaced by 'certain'. Consequently, the evaluation of the prediction procedure could be a matter of comparing just predicted max/min values to the corresponding measured $\max / \min$ values for given prediction sequences. However, as will be addressed in the remaining sections, the evaluation is conducted significantly more thorough.

\subsection{Kriging vs. non-Gaussian processes}

It turns out ${ }^{1}$ that Eq. (14) can be derived also from Kriging which is a statistical regression and/or prediction method, where the basic idea is to predict the value of a function at a given point by computing a weighted average of the known values of the function in the neighborhood of the point [25, 26, 27, 28]. Thus, the resemblance to the presented prediction procedure is clear.

As such, the derivations of the 'Kriging equations' do not need a specific assumption about (multivariate) Gaussian processes, although some authors will

\footnotetext{
${ }^{1}$ Thanks to an anonymous reviewer.
} 
claim that, in practice, this is necessary and why the Kriging models often are referred to a Gaussian process models [29]. Briefly said, the assumptions are 1) Stationarity and 2) Isotropy. However, one limitation is that, in general, the accuracy of interpolation by Kriging will be limited if the number of sampled observations is small, e.g. [26]. Consequently, the Gaussian assumption is implicitly imposed, because of the Central Limit Theorem.

Kriging will not be explored any further in the present article but, obviously, it should be interesting, as a future work, to look closer into Kriging to examine the method for potential use in the context of short-time, deterministic prediction of wave-induced processes. Some useful references can be found on the general topic of Kriging in relation to marine and offshore applications by consulting $[30,31]$. To close the discussion about Kriging, and with given knowledge at hand, it appears that the Gaussian assumption can be relaxed, as Eq. (14) can be found by 'Simple Kriging'. Nonetheless, in the work by Lindgren [23], which is the original reference for the present work including Eq. (14), the assumption is a Gaussian process, and therefore the Gaussian assumption is kept herein.

\section{Experimental data}

\subsection{Testing facility}

The prediction procedure has been applied to experimental model-scale data obtained in a testing facility at the Marine Cybernetics Laboratory (MCLab) at the Norwegian University of Science and Technology (NTNU), Trondheim. The facility includes a basin with dimensions $40 \mathrm{~m} \times 6.45 \mathrm{~m} \times 1.41 \mathrm{~m}(L \times B \times D)$, a vision-based positioning system that provides position and orientation measurements of a dynamic positioned (DP) vessel, and a wave flap ${ }^{2}$ for generating long-crested waves derived from a given wave spectrum. Figure 2 shows the specific model, Cybership 3, in action. The particular ship is a 1:30 scale model

\footnotetext{
${ }^{2}$ DHI Wave Synthesizer, www.dhigroup.com.
} 

system, operator laptop and cRIO is via Ethernet.
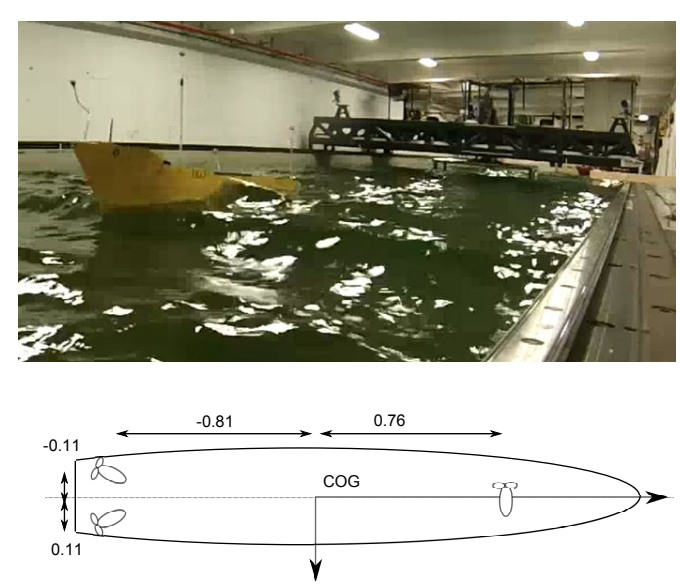

Figure 2: Cybership 3 deployed in the model-basin at NTNU (top), and thruster configuration of the vessel (bottom) with measures in meters. [32]

of a platform supply vessel with dimensions $L_{p p}=1.97 \mathrm{~m}$ and $B=0.44 \mathrm{~m}$. It is equipped with three azimuth thrusters; two at the stern with fixed angles of $\pm 30^{\circ}$ and one in the bow at $90^{\circ}$ (Fig. 2). The vessel has eight $12 \mathrm{~V}$ batteries supplying power to the thrusters and a National Instruments ${ }^{\mathrm{TM}}$ CompactRIO (cRIO) that runs the DP control system. The operator supplies setpoints and specifies controller-gains from a laptop, and communication between the camera

\subsection{Experiments and motion measurements}

The experimental tests have been run with Cybership 3 exposed to different wave scenarios; in each case with the (irregular) sea state specified in terms of a parameterised wave spectrum that has as input significant wave height $H_{s}$ and peak period $T_{p}$. The tests are made with different (relative) wave headings $\beta$, and a summary of the experimental conditions are given in Table 1. All tests are made at zero-forward speed, and with the experimental conditions fixed for the single test case; since the prediction procedure requires/assumes stationary conditions (Section 2). The specific wave heading, used in subcases 'a', 'b' or ' $c$ ', is given in the parenthesis $\{\ldots\}$, where $\beta=0^{\circ}$ is head sea (and $\beta=180^{\circ}$ 
Table 1: Experimental conditions of the test cases. Note, conditions apply to model scale.

\begin{tabular}{lcccc}
\hline Case no. & Spectrum & $H_{s}[\mathrm{~m}]$ & $T_{p}[\mathrm{~s}]$ & $\beta\left[^{\circ}\right]$ \\
\hline 1a,b,c & JONSWAP & 0.04 & 0.8 & $\{0,10,20\}$ \\
2a,b,c & JONSWAP & 0.05 & 0.9 & $\{0,10,20\}$ \\
3a,b,c & JONSWAP & 0.05 & 1.5 & $\{0,10,20\}$ \\
4a,b,c & Ochi-Hubble & $(0.04+0.04)$ & $(0.8+1.5)$ & $\{0,10,20\}$ \\
\hline
\end{tabular}

is following sea). It is decided to keep data in model scale throughout; this includes all analyses and associated results.

The use of long-crested wave presumably does not influence the outcome of predictions, neither positively nor negatively, as the resulting stochastic properties of the wave-induced process, i.e. the motion of the vessel response, are unaffected. It should, however, be interesting to examine this hypothesis closer by conducting model-scale (or full-scale) experiment in short-crested seas. In the same line, it will be of no (theoretical) importance whether the ship advances with a constant forward speed or is at zero-forward speed, as the wave-induced process is stationary in either case; obviously, taking all other experimental conditions/parameters as constant too.

From Table 1 it is seen that totally 12 (sub)cases are investigated. For each subcase, the components of the six degrees-of-freedom motion of Cybership 3 have been measured and corresponding time history records thus exist. Onwards, it is chosen to focus almost entirely on the heave recordings, although analyses have been also made with roll and pitch; but leaving just a few comments, here and there, on these motion components. It is important to note that, in all of the considered cases, approximately ten minutes of stationary motion recordings are available. For the given sea states, noting the values of associated wave periods $T_{p}$ (Table 1 ), 10-minutes recording lengths imply that the vessel encounters about 400-700 single waves, depending on the case $\left(T_{p}\right)$ in study. The motion recordings were initially sampled at $100 \mathrm{~Hz}$ but, as a post-process, data has been resampled to $20 \mathrm{~Hz}$. The reason to down-sample is merely a matter of saving memory/storage on the authors' personal computers, and increase computational efficiency as reduced sampling frequency leads to 
smaller dimension of the autocorrelation matrix. Down-sampling to $20 \mathrm{~Hz}$ will not affect any of the global wave-induced responses; not even in model-scale (1:30).

\subsection{Pre-analysis of measurements data}

One example of a heave recording is shown in Figure 3, which shows both the time history recording and the corresponding periodogram, i.e. the response spectrum, of Case 1a. The response spectrum is shown without and with smoothing (legends 'No smoothing', respectively ' $L=2,400$ ' and ' $\mathrm{L}=$ 200') where smoothing is applied using a Parzen window on the estimated autocovariance function. In practice, the spectral calculation has been made with WAFO [33], and in this case (Fig. 3) the smoothing window functions have 2,400 and 240 elements/weights. ${ }^{3}$ The one value, $\mathrm{L}=2,400$, is equivalent to one fifth of the total number of samples in the particular time history recording. It is noteworthy that this amount of smoothing is used throughout the

${ }^{3}$ In WAFO, the size of the smoothing window is controlled by parameter $\mathrm{L}$.
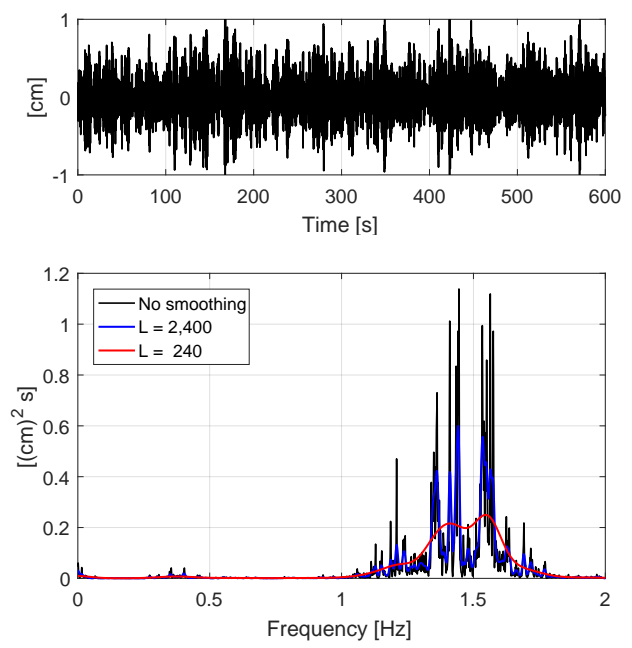

Figure 3: Time history recording (top) and corresponding response spectrum (bottom) with three versions of the spectrum; without and with smoothing controlled by the parameter ' $L$ '. 

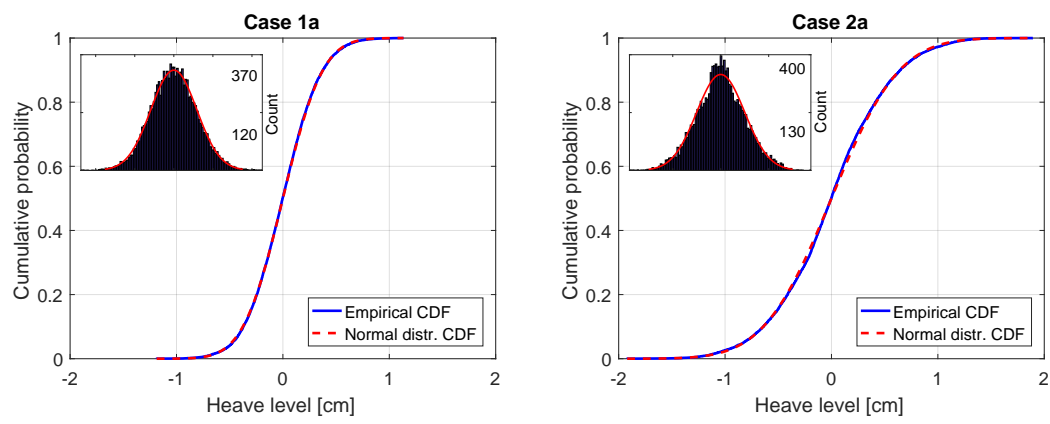

Figure 4: Cumulative distribution functions and corresponding histograms ("internal" plots) of the heave level of Cases 1a and 2a.

forthcoming analyses; however, with no special attempt to "justify" the value $\mathrm{L}=2,400$, although some further remarks on smoothing and its consequence are given in Subsection 4.1.

Mathematically, the prediction procedure assumes, or requires, data to be normal distributed; which for most wave-induced (global) vessel responses typically is a reasonable assumption. Two visual evaluations of the (model-scale) data studied herein are shown in Figure 4, where the two plots apply to the time history recordings of cases 1a and 2a. The plots show the empirical cumulative distribution function ( $\mathrm{CDF}$ ) together with a cumulative normal distribution function having mean value and standard deviation as calculated from the empirical data of the single case. Additionally, each plot presents, as the smaller plot inside, a histogram of the empirical distribution including a fitted normal probability density function. It is seen that the considered cases, 1a and 2a, seemingly represent a normal distributed process, and albeit not shown herein similar findings/visualisations apply to all the other cases listed in Table 1. The visual evaluation can be supplemented with a quantitative/objective test using hypothesis testings, e.g. Anderson-Darling, Kolmogorov-Smirnov, and Lilliefors $[34,35]$, where data is tested against the null hypothesis $[36,37]$ that it follows some pre-specified distribution; or the alternative that data does not follow the specific distribution. The outcome of a hypothesis test is usually a logical, 1 or 0 , where ' 1 ' indicates that the hypothesis is rejected, and ' 0 ' means that 
the test fails to reject the hypothesis. The result, 1 or 0 , is based on the test statistics, considered as a metric/distance $A^{2}$, relative to a certain significance level $[38,39]$. In the present context 'rejection' thus implies that data is not normally distributed, and the alternative means it $i s$. For the specific cases in Figure 4, the data sets have been tested using the Anderson-Darling test ${ }^{4}$ with a $5 \%$ significance level, and it is interesting to note that the data sets, i.e. the time history recordings, of Cases 1a and 2a are not normally distributed. Therefore, it will be interesting to see if predictions, on average, behave differently in terms of agreement relative to measurements, depending on the underlying probability distribution of the data.

The Anderson-Darling test has been applied to all time history recordings, and the result can be seen in Table 2 which specifies whether data follows a normal distribution or, the alternative, that it does not; with values 'Yes' or 'No', respectively, in the specific column. The decision is, as mentioned, based on the test statistics $A^{2}$ relative to a $5 \%$ significance level where the latter, for the given time history recordings, directly translates into an associated critical value $c_{V}$. Thus, data is stationary if $A^{2}<c_{V}$, equivalently $c_{V}-A^{2}>0$, and otherwise data is not, and Table 2 yields also the relative deviation $\frac{c_{V}-A^{2}}{c_{V}}$ to indicate the "degree of normality", or the opposite.

Additionally, Table 2 presents a summary of a pre-analysis made on the measurements data. Thus, the table provides some of the (spectral) parameters characterising the time history recordings; this includes the standard deviation $\sigma$, the mean zero-upcrossing period $T_{z}$ and the spectral bandwidth parameter $\varepsilon$. These parameters can all be calculated using the spectral moments (cf. Eq.

\footnotetext{
${ }^{4}$ The actual computation is performed using the built-in function adtest of MATLAB ${ }^{\circledR}$
} 


$$
\begin{aligned}
\sigma & =\sqrt{m_{0}} \\
T_{z} & =\sqrt{\frac{m_{0}}{m_{2}}} \\
\varepsilon & =\sqrt{1-\frac{m_{2}^{2}}{m_{0} m_{4}}}
\end{aligned}
$$

429

Finally, as the rightmost column in Table 2, the result of another hypothesis test is shown. This test looks into whether data can be considered as stationary or not, and is based on the outcome of an 'Augmented Dickey-Fuller test' which is used to indicate rejection of the presence of a unit root or failure to reject one in the given time history recording. ${ }^{5}$ Herein, it is understood that the presence of a unit root implies that data is non-stationary (and may have a trend). The principle of the test is much the same as the test for a normal distribution, which means that the actual outcome relies on some test statistics. Contrary to the test for a normal distribution it is, however, found that all time history recordings can be considered as stationary and no further remarks are given.

\section{Results and discussions}

The experimental data, including the pre-analysis, described in the previous section will be used to evaluate the prediction procedure outlined in Section 2. The evaluation will be focused on merely the outcome of the prediction procedure when specific settings are applied. This leaves out any sensitivity and parameter studies in the following analysis. On the other hand, the work by Nielsen and Jensen [22] has some detailed studies in this respect for what reason 'guidance' from [22] is indeed valuable.

\subsection{Prediction settings}

In the particular work [22], studies were made on the prediction settings and their influence on any prediction. Specifically, efforts looked at the conse-

${ }^{5}$ The actual test is performed using adftest of MATLAB ${ }^{\circledR}$ without augmented difference terms. 
Table 2: Spectral parameters of the underlying time history recordings and results of hypothesis testings with regards to a normal distribution and stationarity, respectively.

\begin{tabular}{cccccc}
\hline Case & $\sigma[\mathrm{cm}]$ & $T_{z}[\mathrm{~s}]$ & $\varepsilon[-]$ & Normally distr. & Stationary \\
\hline 1a & 0.29 & 0.62 & 0.90 & 'No' (-0.28) & 'Yes' \\
1b & 0.25 & 0.62 & 0.90 & 'Yes' $(0.21)$ & 'Yes' \\
1c & 0.26 & 0.64 & 0.90 & 'No' (-3.87) & 'Yes' \\
2a & 0.50 & 0.74 & 0.91 & 'No' (-5.33) & 'Yes' \\
2b & 0.42 & 0.65 & 0.90 & 'No' (-2.25) & 'Yes' \\
2c & 0.42 & 0.67 & 0.91 & 'Yes' $(0.53)$ & 'Yes' \\
3a & 0.80 & 0.94 & 0.96 & 'No' (-0.97) & 'Yes' \\
3b & 0.75 & 0.92 & 0.96 & 'Yes' $(0.35)$ & 'Yes' \\
3c & 0.81 & 0.93 & 0.95 & 'Yes' (0.16) & 'Yes' \\
4a & 0.64 & 0.83 & 0.94 & 'Yes' (0.43) & 'Yes' \\
4b & 0.62 & 0.79 & 0.94 & 'Yes' (0.20) & 'Yes' \\
4c & 0.69 & 0.82 & 0.94 & 'Yes' (0.57) & 'Yes' \\
\hline
\end{tabular}

quence in applying different "amounts" of prior data, e.g. to consider the past 10 seconds versus 20 seconds of data, relative to current time $t_{0}$, for making predictions, say, 50 seconds ahead of time $t_{0}$; with all times in full-scale. Moreover, the importance of settings related to the spectral calculation of the sample autocorrelation function (ACF) was addressed, since smoothing, as discussed in Section 3, affects significantly the shape of the periodogram from which the sample ACF is derived. The most "complete" sample ACF is obtained when no smoothing is applied to the periodogram, and, in this case, the spectral-version of the sample ACF will be identical to the sample ACF as if computed directly according to its definition in the time domain (Eq. 10). However, it is also known that for zero-smoothing, the sample autocorrelation function may fail to damp out according to expectation $[21,40]$. Consequently, correlation may appear to last (be present) for longer duration than is actually true, and some smoothing is therefore necessary. On the other hand, if too much smoothing is applied to the periodogram, correlation will appear to vanish after only a short time, or equivalently said the sample ACF damps out too quickly.

Previously, it was explained that, in the present study, smoothing is applied to data using a Parzen window on the estimated autocovariance function and, 
hence, contributions from covariance at large lags, which are generally not reliable, will be small or zero. Three versions of sample ACFs, all obtained from exactly the same data, are visualised in Figure 5; with the underlying time history recording and the amounts of smoothing identical to what was studied previously (Fig. 3). Indeed, it is seen how varying degrees of smoothing may affect the sample ACF very much. Consequently, a prediction procedure relying fundamentally on the sample ACF will be influenced by the degree of smoothing being applied in the spectral calculations. Nonetheless, the conclusions drawn from the earlier study by Nielsen and Jensen [22], made on a very large set of numerical time history simulations, suggest, or "prescribe", that predictions, relative to corresponding measurements, are improved by taking into account correlation/autocovariance at large lags despite they are not necessarily always ("mathematically") reliable.

In summary, predictions will be made with the following settings, cf. Nielsen and Jensen [22], which apply to model scale:

- Predictions ahead of current time $t_{0}$ take into account $N$ past measurement points (relative to $t_{0}$ ), where the value of $N$ is equivalent to a time period $T_{\text {past }}=25 T_{p}$ with $T_{p}$ given in Table 1.

- The periodogram is smoothed using a Parzen window on the estimated autocovariance function. The window function is of size $\mathrm{L}$, where $\mathrm{L}$ is taken as one fifth of the total number of samples in the particular time

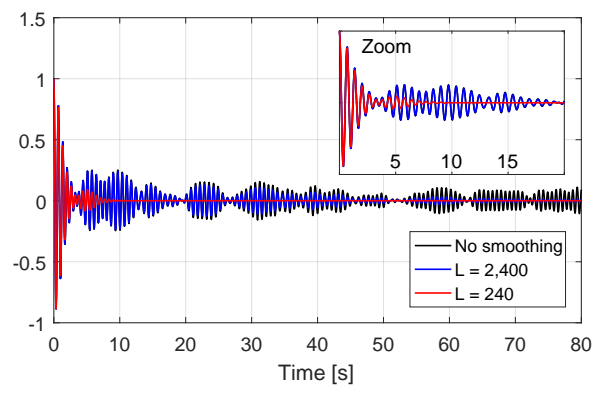

Figure 5: Sample autocorrelation function with a zoom included as the "internal" plot. The underlying time history recording is as seen in Figure 3. 

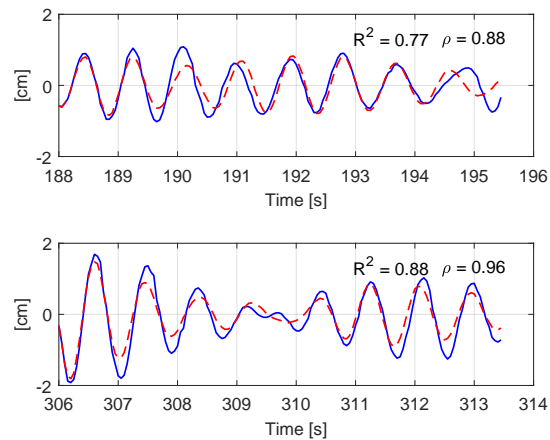
depending on the case $\left(T_{p}\right)$ in study. prediction sequences overlap each other.

\subsection{Visual and statistical comparisons}

history recording in study.

- Finally, predictions will be made 7.5 seconds ahead of any current time $t_{0}$, and a new prediction is made every 2.0 second on the 10-minutes time strips. It is noted that 7.5 seconds correspond to about $5-8$ wave periods,

These settings are applied to all time history recordings, cf. Tables 1 and 2. On each 10-minutes time history strip, totally 200 prediction sequences have been computed; taking note that the initial 50 seconds and the last approximately 90 seconds of any recording are not considered, and remembering also that

Figure 6 shows four prediction sequences; all made for data strips taken from Case 2a. The plots include the measured (i.e., the true) response sequences, and, furthermore, two (statistical) numbers, $R^{2}$ and $\rho$, are printed in the upper right corner of each plot. Leaving the two numbers to be defined and explained later, the particular plots reveal good agreement between the predicted and measured response sequences on almost the entire part of the individual data strips. However, generally it is not every prediction sequence of Case 2a which agrees as
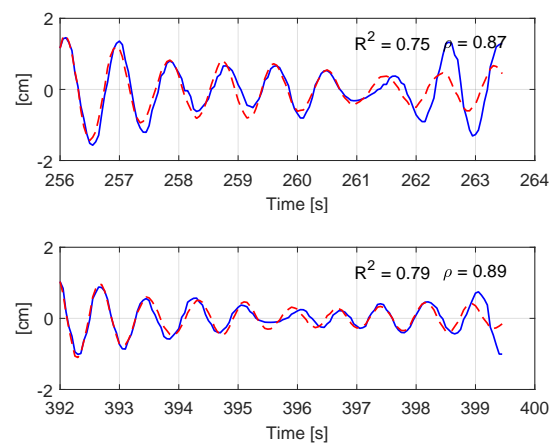

Figure 6: Selected heave response sequences of Case 2a; blue full line is measurement and dashed red line is prediction. The determination and correlation coefficients, $R^{2}$ respectively $\rho$ (defined later), are seen in the upper right-hand corner. 


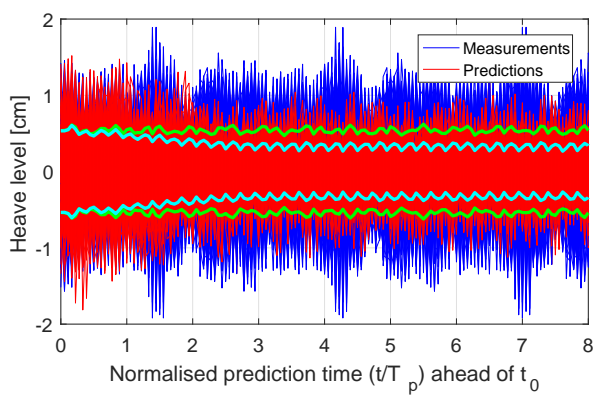

Figure 7: Entire set of prediction and measurement sequences of Case 2a; noting that there are $2 \times 200$ curves on top of each other. Additionally, the pairs of bold lines in cyan (predictions) and green (measurements) indicate the 'point-wise' standard deviation calculated from the $2 \times 200$ data points at given time instants.

accurate to the measured corresponding one, as seen from the plots/comparisons in Figure 6. Therefore, to get a better, or more "average", picture of the overall performance of the prediction procedure, a random excerpt of visual comparisons can be seen in Figures A.13-A.15 in Appendix A, which presents sets of plots similar to those in Figure 6. The sets seen in Figures A.13, A.14, A.15 apply to Cases $2 \mathrm{a}, 2 \mathrm{~b}$, and $2 \mathrm{c}$, respectively, and the excerpts (of prediction vs. measurement sequences) are simply based on 16 data strips, for each case, cut out every 20 second starting at $t_{0}=50 \mathrm{~s}$. Thus, the average performance of the prediction procedure is better evaluated since no special focus is on "good" predictions, nor "bad" ones.

On the other hand, it is not practically possible, nor feasible, to visually compare - for all data sets (Cases 1-4), cf. Section 3 - every prediction sequence with the corresponding measurement sequence in single and detailed plots like studied in, e.g., Figures 6 and A.13-A.15. Therefore, to derive some sort of 'statistical measure' of the average performance of the prediction procedure, another presentation of the outcome/comparison is studied. Collectively, Figure 7 shows the results of all heave prediction sequences and the corresponding measurement sequences of Case 2a. The abscissa represent the normalised prediction time ahead of $t_{0}$, where normalisation is made with respect to the wave peak period $T_{p}$ (cf. Table 2). In addition to all pairs (prediction vs. mea- 


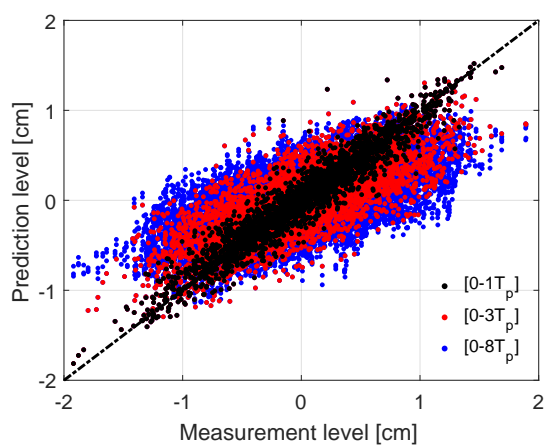

Figure 8: Agreement between single pairs of measurements and predictions for Case $2 a$ with separate results depending on the prediction horizon considered; e.g., $\left[0-3 T_{p}\right]$ where $T_{p}$ is the wave peak period.

surement) of data sequences, the curves of the point-wise standard deviation are included as pairs of plus/minus versions of it; shown as the green and cyan pairs of bold lines for measurements and predictions, respectively. Thus, keeping in mind, there are $2 \times 200$ prediction and measurement points at any instant, where the individual set of points has a mean value of zero and a standard deviation as visualised in Figure 7. From the plot in Figure 7, it is noted that until about $1 T_{p}$ ahead of current time $t_{0}$ there is a very good agreement between predictions and measurements. Subsequently, the agreement reduces and at prediction instants from about $3 T_{p}$ and further ahead until the end (taken as $\left.8 T_{p}\right)$, the agreement remains, on average, at the same level. These findings are confirmed by the plot in Figure 8 which presents the agreement between any single pair of data values (prediction vs. measurement) obtained for Case 2a; with specified results dependent on the prediction horizon: $\left[0-1 T_{p}\right],\left[0-3 T_{p}\right]$ and $\left[0-8 T_{p}\right]$, respectively. Moreover, the theoretical line of perfect agreement is included in the plot as the black dashed line.

Appendix B contains pairs of plots equivalent to those in Figures 7 and 8 but considering subcases $2 \mathrm{a}, 2 \mathrm{~b}$, and $2 \mathrm{c}$ together. Generally, the observations from the (other) subcases, see Figures B.17 and B.18 in the appendix, are similar to what was addressed above, although the agreements for Cases 
$2 \mathrm{~b}$ and $2 \mathrm{c}$ reduce to slightly lower levels than found for Case 2a. One particular "characteristic", evident from all cases (2a-c), is the decreasing amplitude levels, equivalently decreasing point-wise standard deviations, for prolonged prediction horizon/interval of the prediction sequences. This observation can be explained mathematically, cf. Lindgren [23], since the predicting process (Eq. 14) is non-stationary with properties resembling the autocorrelation function of a (stochastic) wave-induced process.

As a last visual comparison, see Figure 9, the relative error between corresponding set of heave sequences (prediction versus measurement), as seen in Figure 7, has been calculated for Case 2a; where normalisation is made with respect to the square root of the 0th-order spectral moment, cf. Table 2. The error curves for all corresponding sequences are shown in Figure 9 as the blue (thin) lines. Notably, the plot sheds light on four specific error curves (coloured in green); namely, those four obtained by considering the prediction and measurement sequences shown in Figure 6. Furthermore, the plot in Figure 9 includes the point-wise mean value curve and the ditto curves of plus/minus the pointwise standard deviation (StD) of the errors, where the former curve fluctuates around zero as expected. It is interesting to observe that even for sequences like those studied previously (Figure 6), where the agreement, based on a visual judgement, is apparently very acceptable, still the relative, normalised error is not insignificant; taking note that the four green curves in the plot in Figure 9 exceed the standard deviation of the point-wise error at several instants. Consequently, a large number of the prediction sequences seen in Figure 7 reveals just as good, or better, "visual agreement" as what can be seen from the four individual plots in Figure 6.

Obviously, the various plots like those discussed above are visual indicators of the general performance of the prediction procedure. Nonetheless, the discussion(s) can be supplemented with some quantitative error measures and/or statistical evaluations. In this context, it should be clear that some metric(s) must be computed to comprehensively evaluate the overall performance of the prediction procedure, since any visual comparison that can be made from the 


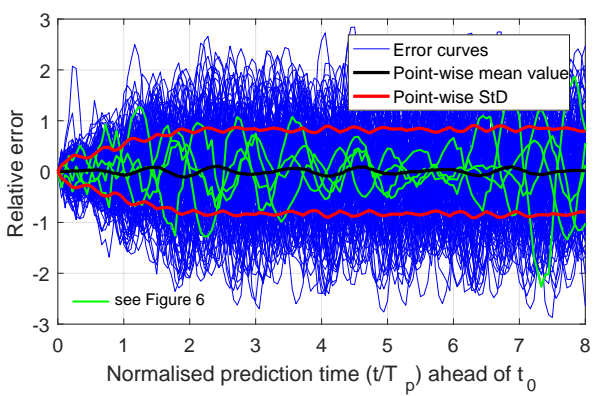

Figure 9: Normalised error between prediction and measurement for all sequences of Case $2 a$.

plots in, for instance, Figures 6 and 8 always will be, to some degree, rather subjective. On the other hand, it is not straight forward to define unique and physically meaningful metrics to make comparisons from. This topic has been discussed in several of other similar works, e.g. [41, 18, 13, 22], and the matter is, to some extent, an entire topic in its own right. In the present study, attention is given to two metrics; the one taken as the Pearson Correlation Coefficient, $\rho$, and the other taken as the Determination Coefficient, $R^{2}$, defined by, respectively,

$$
\begin{aligned}
\rho & =\frac{\sum_{i=1}^{N}\left(\widehat{x}_{i}-\mu_{\widehat{x}}\right)\left(x_{i}-\mu_{x}\right)}{\sqrt{\sum_{i=1}^{N}\left(\widehat{x}_{i}-\mu_{\widehat{x}}\right)^{2}} \sqrt{\sum_{i=1}^{N}\left(x_{i}-\mu_{x}\right)^{2}}} \\
R^{2} & =1-\frac{\sum_{i=1}^{N}\left(\widehat{x}_{i}-x_{i}\right)^{2}}{\sum_{i=1}^{N}\left(x_{i}-\mu_{x}\right)^{2}}
\end{aligned}
$$

Here, the former is a direct measure of the linear dependence (correlation) between the two sequences; the prediction sequence, $\widehat{\mathbf{x}}$, and the corresponding measurement sequence, $\mathbf{x}$, on a data strip with totally $N$ pairs of observations $\left\{\widehat{x}_{i}, x\right\}$ with mean values $\left\{\mu_{\widehat{x}}, \mu_{x}\right\}$ on the specific data strip. The correlation coefficient $\rho$ is 1 for perfect correlation, -1 for anti-correlation, and $-1<\rho<1$ for anything in between. The determination coefficient $r$-squared indicates to some degree the 'goodness of fit', on average, for the pairs of observations. It has a value of 1 if the fit is perfect, and otherwise $R^{2}<1$.

Although other metrics could be considered, see Nielsen and Jensen [22], 
these two metrics have, in their combined use, the potential to quantitatively assess the agreement between predictions and measurements. It is noteworthy that some studies in the literature focus on only correlation as the measure for comparison. However, in principle, a correlation coefficient by itself has little meaning, if not the actual values of measurement and prediction are close to each other at any given observation point $i$. Therefore, it is necessary to introduce also a measure of the agreement between individual observations (prediction vs. measurement) for what reason the determination coefficient $R^{2}$ is used together with $\rho$. It is noteworthy that in this particular context, the r-squared value can lie outside $[0 ; 1]$ with negative values; which is usually not the case for an r-squared value when the coefficient is calculated/applied in regression analysis $[42,43]$. The issue here is that the determination coefficient, in the present application, is used in a different way than what is the typical way in (linear) regression analysis, where a regression model is fitted to data, so that the value of the coefficient is a measure of how well observed outcomes are replicated by the regression model itself, based on the proportion of total variation of outcomes explained by the model, cf. [44].

The correlation coefficient and the determination coefficient have been computed for every sequence (200 in total) within each of the test cases, cf. Table 1, and specific outcomes of the coefficients are included in Figure 6, where the values of $\rho$ and $R^{2}$ are seen in the upper right-hand corner of each plot. Likewise, the values of the metrics appear in the plots of the sequences visualised in Appendix A. If focus is turned on all the sequences of Case 2a, the result is presented in Figure 10, and it is clear that the two coefficients, $\rho$ and $R^{2}$, show some variation with both higher and lower values, indicating sequences with good agreement and the opposite, respectively, between predictions and measurements. The similar plots of Cases $2 \mathrm{~b}$ and $2 \mathrm{c}$ have been included in Appendix C.

Table 3 presents the statistics of all cases, including results of roll and pitch, with the mean value and the coefficient of variation $(\mathrm{CoV}=$ "standard dev./mean") noted for the correlation coefficient and the determination coeffi- 
Table 3: Statistics, i.e. mean values, of the correlation coefficient $\rho$ and the determination coefficient $R^{2}$, respectively, with results for heave, roll, and pitch. Note, the coefficient of variation (CoV) is included in parenthesis.

\begin{tabular}{|c|c|c|c|c|c|c|}
\hline \multirow[b]{2}{*}{ Case } & \multicolumn{2}{|l|}{ Heave } & \multicolumn{2}{|l|}{ Roll } & \multicolumn{2}{|l|}{ Pitch } \\
\hline & $\rho[-]$ & $R^{2} \quad[-]$ & $\rho[-]$ & $R^{2} \quad[-]$ & $\rho[-]$ & $R^{2} \quad[-]$ \\
\hline $1 \mathrm{a}$ & $0.54(0.50)$ & $0.26(1.22)$ & $0.67(0.33)$ & $0.43(0.74)$ & $0.42(0.65)$ & $0.17(1.32)$ \\
\hline $1 b$ & $0.48(0.56)$ & $0.21(1.34)$ & $0.53(0.52)$ & $0.27(1.10)$ & $0.50(0.50)$ & $0.24(1.12)$ \\
\hline $1 \mathrm{c}$ & $0.42(0.56)$ & $0.15(1.52)$ & $0.50(0.55)$ & $0.24(1.29)$ & $0.46(0.53)$ & $0.19(1.37)$ \\
\hline $2 \mathrm{a}$ & $0.67(0.29)$ & $0.44(0.59)$ & $0.62(0.40)$ & $0.35(0.98)$ & $0.42(0.51)$ & $0.17(1.18)$ \\
\hline $2 \mathrm{~b}$ & $0.56(0.45)$ & $0.31(0.91)$ & $0.62(0.46)$ & $0.36(0.93)$ & $0.47(0.48)$ & $0.21(1.10)$ \\
\hline $2 c$ & $0.40(0.67)$ & $0.16(1.55)$ & $0.55(0.62)$ & $0.30(1.24)$ & $0.47(0.70)$ & $0.22(1.42)$ \\
\hline $3 a$ & $0.54(0.42)$ & $0.28(0.94)$ & $0.65(0.36)$ & $0.40(0.82)$ & $0.49(0.46)$ & $0.24(1.01)$ \\
\hline $3 \mathrm{~b}$ & $0.53(0.51)$ & $0.26(1.25)$ & $0.61(0.47)$ & $0.35(1.00)$ & $0.55(0.45)$ & $0.28(0.96)$ \\
\hline $3 c$ & $0.53(0.45)$ & $0.28(0.88)$ & $0.61(0.40)$ & $0.35(0.92)$ & $0.54(0.42)$ & $0.29(0.76)$ \\
\hline $4 a$ & $0.48(0.40)$ & $0.23(0.83)$ & $0.49(0.39)$ & $0.32(0.96)$ & $0.45(0.45)$ & $0.20(0.93)$ \\
\hline $4 \mathrm{~b}$ & $0.48(0.39)$ & $0.21(0.95)$ & $0.58(0.45)$ & $0.30(1.18)$ & $0.44(0.45)$ & $0.17(1.18)$ \\
\hline $4 c$ & $0.48(0.43)$ & $0.23(0.85)$ & $0.54(0.58)$ & $0.27(1.36)$ & $0.47(0.41)$ & $0.21(0.96)$ \\
\hline Average & 0.51 & 0.25 & 0.58 & 0.33 & 0.47 & 0.22 \\
\hline
\end{tabular}

cient, respectively.

It can be argued that the results presented in Table 3, i.e. the determination and the correlation coefficients, have the most meaning when they are discussed in relative terms and not considered as absolute statistical measures

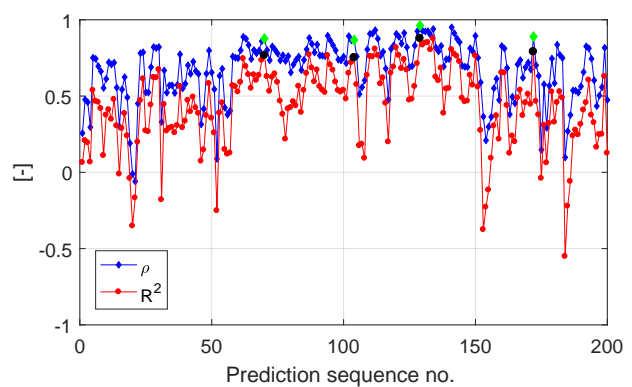

Figure 10: Determination coefficients $R^{2}$ (red) and correlation coefficients $\rho$ (blue) of Case 2a. The results corresponding to the sequences from Figure 6 are indicated by the larger marker sizes in colours black and green in contrast to red and blue, respectively. 
and/or performance indicators. Thus, the coefficients should be rather used as relative indicators of the performance of the prediction procedure, when this is applied under different, but specific, settings and to various, but similar, experimental conditions (including vessel type, sea state, motions/responses, etc.). For instance, Table 3 suggests that heave, on average, may be predicted most accurately when the vessel faces the waves head sea (subcases 'a'), since the correlation coefficient and the determination coefficient consistently attain the highest average values in these cases; compared to headings off head sea (subcases 'b' and 'c'). An almost similar finding is observed for roll but not for pitch. The table also reveals that roll of the three responses, for the considered ship and sea states, can be predicted with the best accuracy. Albeit not shown (directly), it is in itself interesting that significant roll is actually induced even when the heading is straight head sea (and also slightly off). The physical explanation may be that some wave reflection occurs from the tank wall sides, and/or the explanation may be because of the DP system. This issue is, however, not considered any further in the present study but another should try to resolve the "problem".

Previously, all the time history recordings were tested for their probability distribution to be of a normal distribution type, cf. Table 2. It is interesting to note from Table 3 that it does not seem to be of any role whether the data is normally distributed or not, when the (average) agreement between prediction and measurement sequences are studied. Thus, the best results for heave are found for Cases 2a and 2b, where data - according to the Anderson-Darling test - should not be considered to be normally distributed. It is therefore of no fundamental importance that data, in practice, follows a normal distribution, despite the theoretical formulation of the prediction procedure assumes that data originates from a normal distributed process, cf. Lindgren [23].

Another way to make use of the correlation coefficient and the determination coefficient is to study their behaviour with the prediction horizon (ahead of current time). This sort of analysis can be used to evaluate, in relative terms, when predictions statistically will be less reliable. Table 4 presents the result of 
Table 4: Heave statistics: Behaviour of the correlation coefficient $\rho$ and the determination coefficient $R^{2}$ with prediction horizon ahead of $t_{0}$. Note, the coefficient of variation (CoV) is included in parenthesis.

\begin{tabular}{|c|c|c|c|c|c|c|}
\hline \multirow[b]{2}{*}{ Case } & \multicolumn{2}{|l|}{$[0-2] \mathrm{s}$} & \multicolumn{2}{|l|}{$[0-4] \mathrm{s}$} & \multicolumn{2}{|l|}{$[0-6] \mathrm{s}$} \\
\hline & $\rho[-]$ & $R^{2} \quad[-]$ & $\rho[-]$ & $R^{2} \quad[-]$ & $\rho[-]$ & $R^{2} \quad[-]$ \\
\hline $1 \mathrm{a}$ & $0.72(0.46)$ & $0.42(1.55)$ & $0.59(0.57)$ & $0.31(1.68)$ & $0.55(0.53)$ & $0.28(1.38)$ \\
\hline $1 b$ & $0.66(0.50)$ & $0.36(1.55)$ & $0.53(0.59)$ & $0.25(1.66)$ & $0.49(0.58)$ & $0.22(1.46)$ \\
\hline $1 \mathrm{c}$ & $0.66(0.45)$ & $0.32(1.69)$ & $0.51(0.53)$ & $0.22(1.56)$ & $0.45(0.56)$ & $0.17(1.46)$ \\
\hline $2 \mathrm{a}$ & $0.80(0.31)$ & $0.53(1.21)$ & $0.72(0.31)$ & $0.47(0.80)$ & $0.69(0.29)$ & $0.45(0.63)$ \\
\hline $2 \mathrm{~b}$ & $0.77(0.32)$ & $0.51(0.92)$ & $0.65(0.40)$ & $0.38(0.96)$ & $0.59(0.43)$ & $0.34(0.89)$ \\
\hline $2 \mathrm{c}$ & $0.70(0.39)$ & $0.43(1.00)$ & $0.53(0.56)$ & $0.28(1.22)$ & $0.44(0.64)$ & $0.19(1.48)$ \\
\hline $3 a$ & $0.78(0.32)$ & $0.53(0.88)$ & $0.66(0.41)$ & $0.38(1.15)$ & $0.58(0.43)$ & $0.32(1.02)$ \\
\hline $3 b$ & $0.80(0.27)$ & $0.53(1.04)$ & $0.66(0.40)$ & $0.38(1.09)$ & $0.57(0.49)$ & $0.29(1.38)$ \\
\hline $3 c$ & $0.78(0.34)$ & $0.58(0.67)$ & $0.65(0.44)$ & $0.41(0.88)$ & $0.57(0.43)$ & $0.32(0.86)$ \\
\hline $4 a$ & $0.69(0.39)$ & $0.44(0.91)$ & $0.57(0.40)$ & $0.32(0.85)$ & $0.51(0.39)$ & $0.26(0.87)$ \\
\hline $4 \mathrm{~b}$ & $0.67(0.40)$ & $0.41(1.15)$ & $0.56(0.43)$ & $0.29(1.12)$ & $0.50(0.42)$ & $0.24(1.06)$ \\
\hline $4 \mathrm{c}$ & $0.69(0.38)$ & $0.41(1.13)$ & $0.57(0.43)$ & $0.31(0.87)$ & $0.51(0.43)$ & $0.26(0.81)$ \\
\hline Average & 0.73 & 0.46 & 0.60 & 0.33 & 0.54 & 0.28 \\
\hline
\end{tabular}

such an analysis made for the heave sequences alone; omitting results of roll and pitch. It is seen that the correlation coefficient and the determination coefficient have been calculated for prediction horizons: $[0-2] \mathrm{s},[0-4] \mathrm{s}$, and $[0-6] \mathrm{s}$. The content of Table 4 has been visulised in Figures 11 and 12, where the data from Table 3 is also included, since this data, of course, represent the full prediction horizon [0-7.5] s. Basically, Table 4 yields a (consistent) quantification of the graphical result presented previously in Figure 8, where the agreement at single time instants with varying prediction horizons was considered for one specific subcase.

Figures 11 and 12 confirm, not surprisingly, what was previously discussed about "reducing" agreement for prolonged prediction horizon. However, it is indeed interesting to see that the largest relative reduction occurs consistently, and for both $\rho$ and $R^{2}$, as the prediction horizon is increased from [0-2] s to $[0-4] \mathrm{s}$, whereas the relative reduction is smaller for the larger horizons. This in- 
dicates that successful predictions, with insignificant reduction in the accuracy, may be obtained for even larger horizons than considered in the present study; leaving the actual investigation for a future study.

\section{Summary and conclusions}

In the article, a procedure facilitating short-time, deterministic prediction of wave-induced vessel responses has been presented. The predicted response sequence applies to a given time horizon in the order 15-60 seconds ahead of current time, and is deterministic in the sense that it is the actual (time-dependent) response oscillation that is computed. The prediction procedure does not need information about the exciting wave scenario; neither in terms of the sea surface elevation nor in terms of a (statistical) wave spectrum. Merely, the procedure requires discretely sampled measurements data of the vessel response to be predicted, so that the only input is the measured time history recording. The procedure is not dependent on off-line training and, thus, predictive calculations
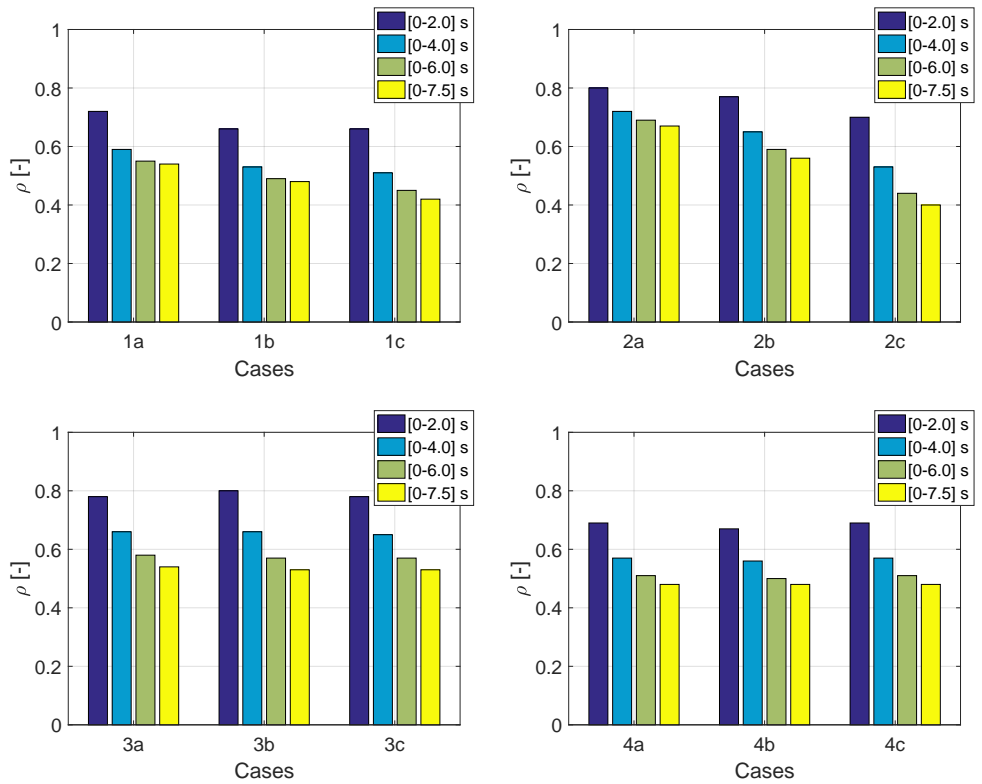

Figure 11: Heave correlation coefficients depending on the prediction horizon. 

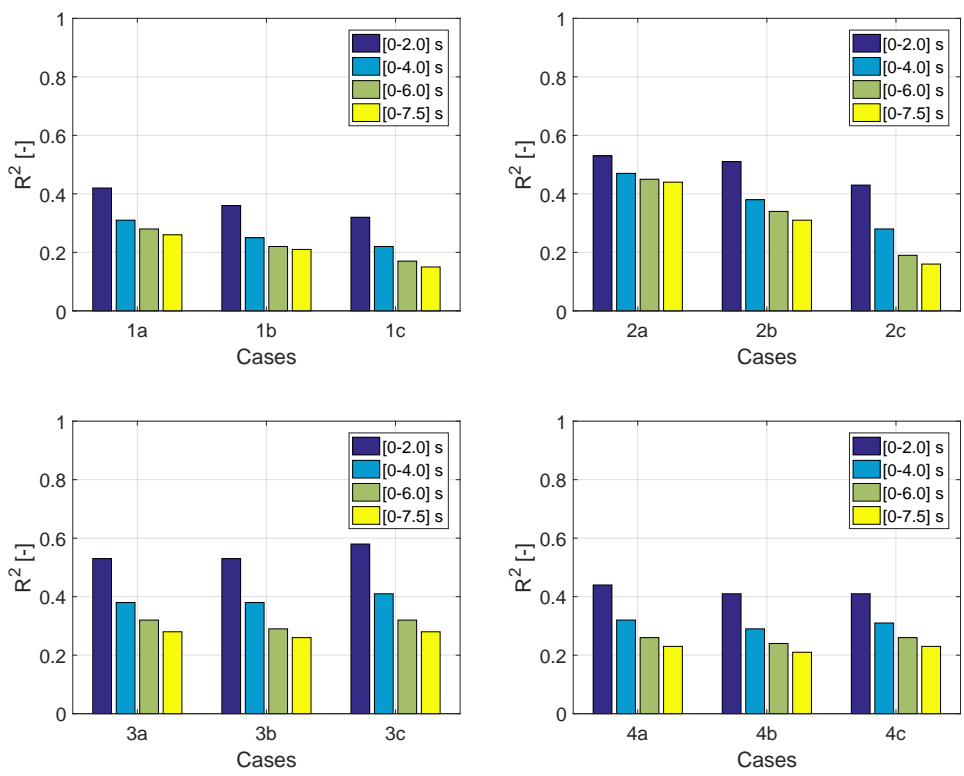

Figure 12: Heave determination coefficients depending on the prediction horizon.

can run real-time. Mathematically, the procedure relies on the observed (measured) sample autocorrelation function of the particular wave-induced response in study. The response is considered to be of a normal distributed process and, in theory, stationary conditions should apply, since the sample autocorrelation function is not reliable otherwise.

The study herein was a direct continuation of earlier studies [20,22] but, for the first time, the prediction procedure has been applied to model-scale data. The experimental data has been obtained from tests conducted at the 'MCLab' at the Norwegian University of Science and Technology, where a 1:30 scale model of a platform supply vessel was exposed to various long-crested, irregular wave scenarios. The main conclusions from the present study do not contradict any of the previous findings in [20,22]. Especially, the following bullets are noteworthy; emphasising that the list not only draws conclusions from work explicitly shown herein but includes also findings from [22] that have been confirmed/elaborated on in the present work without including detailed discussions: 
- Deterministic predictions ahead of time can be made successfully on a given time horizon. In the present study, predictions were computed 7.5 seconds ahead of time. In full scale, this corresponds to a prediction horizon of 41 seconds for the particular vessel. In the tested wave conditions, this time horizon is equivalent to about 8-9 wave periods ahead of current time.

- The accuracy of predictions reduces as the prediction horizon is increased. Generally, for the shorter horizons ahead of time the deviations between prediction and measurement sequences are explained primarily because of a small delay/lag in the prediction. At times further ahead, deviations are present also because the actual values of predictions, at particular instants of time, are off compared to the measured values. This behaviour is seen because the predicted response sequence is non-stationary with properties resembling the autocorrelation function of a (stochastic) waveinduced process.

- The accuracy of the prediction procedure is highly related to the correlation structure of the actual process, as the autocorrelation function is a direct measure of the hydrodynamic memory in the system. Thus, smoothing of the autocorrelation function or, vice versa, the response spectrum will be influencing the outcome of computed predictions.

- Albeit some smoothing must be applied to diminish the influence of covariance contributions at (very) large lags, which are generally not reliable, it is vital to keep some correlation, as the prediction horizon thus is extended.

\subsection{Further work}

The presented work and the associated results show that the considered prediction procedure has the potential to calculate accurately, in real-time, the future wave-induced behaviour of a vessel. This ability will be indeed valuable, 
as it can reduce significantly the probability of failure of many marine operations. Nonetheless, and before the procedure is applied in real-case applications to assist in execution of practical operations, the prediction procedure should be examined further. Thus, it will be relevant to consider some, or all, of the points and/or questions below:

- Previously, the method, as is, has been applied to simulated data [22] and the present article considers model-scale data. This means that stationarity can be taken as a good assumption. It should be useful to examine the procedure with full-scale data; either obtained through dedicated sea trials, or from measurements recorded on an operating vessel, since strictly speaking stationarity does never occur in real-world conditions.

- The effect/influence of smoothing has not been fully explored, and sensitivity studies in this respect will provide useful knowledge. In the same line, it should be tested what is the maximum prediction horizon ahead of time, and what will it depend on; taking that any such 'maximum horizon' exists, i.e. can be calculated. In this context, statistical metrics/measures of the goodness-of-fit needs to be explored.

- Why are predictions good, when they are; or conversely, under which conditions are predictions typically not reliable/accurate. Obviously, keeping in mind here that in real-case scenarios it will be just as important to know when a marine operation should not be conducted, as it is to know when the operation (most likely) can be safely conducted.

- The prediction of the response, at any instant ahead of time, is the main objective. However, is it possible to associate some sort of 'limiting envelope' which estimates upper and lower bounds on the actual prediction sequence. In practical applications/exploitations, this sort of knowledge is more useful than knowing that a response may take a given value at a specific time.

- Is it possible to use knowledge of the probability distribution, or other sta- 
tistical properties, of the errors between previously-made sets of prediction and measurement sequences; both "good" ones and "bad" ones.

- Finally, in a more distant future, it could be interesting to set up a comparative study which will evaluate the performance of different (deterministic) prediction procedures, including the ones requiring offline training (e.g., neural networks, autoregressive-procedures).

\section{Acknowledgement}

The work by the first and second authors was supported by the Research Council of Norway through the Centres of Excellence funding scheme, Project number 223254-AMOS.

\section{References}

[1] J. Dalzell, A Note on Short-Time Prediction of Ship Motions, Journal of Ship Research 9 (1965) 118-121.

[2] M. Triantafyllou, M. Bodson, Real Time Prediction of Marine Vessel Motions Using Kalman Filtering Techniques, in: Proc. 14th Offshore Technology Conference, Houston, TX, USA, 1982.

[3] M. Triantafyllou, M. Bodson, M. Athans, Real Time Estimation of Ship Motions Using Kalman Filtering Techniques, IEEE Journal of Oceanic Engineering 8 (1983) 9-20.

[4] M. Sidar, B. Doolin, On the Feasibility of Real-Time Prediction of Aircraft Carrier Motiona t Sea, IEEE Trans. Automatic Control 28 (1983) 350-356.

[5] D. Broome, Application of ship motion prediction II, International Maritime Technology 110 (1998) 135-153.

[6] D. Broome, M. Hall, Application of ship motion prediction I, International Maritime Technology 110 (1998) 77-93. 
[7] J. Chung, Z. Bien, Y. Kim, A Note on Ship-Motion Predictions based on Wave-Excitation Input Estimation, IEEE Journal of Oceanic Engineering 15 (1990) 244-250.

[8] W.-Y. Duan, L.-M. Huang, Y. Han, R. Wang, IRF - AR Model for ShortTerm Prediction of Ship Motion, in: Proc. 25th ISOPE, Kona, HI, USA, 2015 .

[9] P. From, J. Gravdahl, T. Lillehagen, P. Abbeel, Motion Planning and Control of Robotic Manipulators on Seaborne Platforms, Control Engineering Practice 19 (2011) 809-819.

[10] A. Khan, C. Bil, K. Marion, Real-time prediction of ship motion using artificial neural networks, in: Proc. 3rd Massachusetts Institute of Technology Conference on Computational Fluid and Solid Mechanics, Oxford, UK, 2005.

[11] A. Khan, C. Bil, K. Marion, Ship motion prediction for launch and recovery of air vehicles, in: Proc. of MTS/IEEE OCEANS, Washington D.C., USA, 2005 .

[12] A. Khan, C. Bil, K. Marion, M. Crozier, Real Time Prediciton of Ship Motions and Attitudes Using Advanced Prediction Techniques, in: Proc. 24th Congress of Intl. Council of the Aeronautical Science, Yokohama, Japan, 2004 .

[13] P. Naaijen, D. Roozen, R. Huijsmans, Reducing Operational Risks by OnBoard Phase Resolved Prediction of Wave Induced Ship Motions, in: Proc. 35th OMAE, Busan, Korea, 2016.

[14] X. Peng, X. Zhao, L. Xu, Real-time prediction algorithm research of ship attitude motion based on order selection with corner condition, in: Proc. 1st ISSCAA, Harbin, China, 2006.

[15] J. Woodacre, R. Bauer, R. Irani, A review of vertical motion heave compensation systems, Ocean Engineering 104 (2015) 140-154. 
[16] X. Zhao, R. Xu, C. Kwan, Ship-Motion Prediction: Algorithms and Simulation Results, in: Proc. IEEE ICASSP, Montral, Canada, 2004.

[17] J. Borge, R. Gonzáles, K. Hessner, K. Reichert, C. Soares, Estimation of Sea State Directional Spectra by Using Marine Radar Imaging of Sea Surface, in: Proceedings of ETCE/OMAE2000 Joint Conference, ASME, New Orleans, Louisiana, USA, 2000.

[18] T. Hilmer, E. Thornhill, Observations of predictive skill for real-time Deterministic Sea Waves from the WaMoS II, in: Proc. of MTS/IEEE OCEANS, Washington D.C., USA, 2015.

[19] U. D. Nielsen, A concise account of techniques available for shipboard sea state estimation, Ocean Engineering 129 (2017) 352-362.

[20] I. M. V. Andersen, J. Jensen, U. D. Nielsen, Evaluation of Response Prediction Procedures using Full Scale Measurements for a Container Ship, in: Proc. of 12th PRADS, Changwon, South Korea, 2013.

[21] G. Box, G. Jenkins, G. Reinsel, Time Series Analysis, 4th Edition, Wiley, 2008.

[22] U. D. Nielsen, J. Jensen, Deterministic predictions of vessel responses based on past measurements, in: Proc. 27th ISOPE, San Francisco, CA, USA, 2017.

[23] G. Lindgren, Some Properties of a Normal Process near a Local Maximum, Annals of Mathematical Statistics 41 (1970) 1870-1883.

[24] J. J. Jensen, Load and Global Response of Ships, Vol. 4 of Elsevier Ocean Engineering Book Series, Elsevier, 2001.

[25] Wikipedia, Kriging (URL date 07-07-2017).

[26] R. Olea, Geostatistics fo Engineers and Earth Scientists, Kluwer Academic Publishers, 1999. 
[27] N. Le, J. Zidek, Statistical analysis of environmental space-time processes, Springer, 2006.

[28] C. G. L.A. Waller, Applied Spatial Statistics for Public Health Data, John Wiley \& Sons, 2004.

[29] B. Gaspar, A. Teixeira, C. G. Soares, Assessment of the Efficiency of Kriging Surrogate Models for Structural Reliability Analysis, Probabilistic Engineering Mechanics 37 (2014) 24-34.

[30] J. Pradillon, C. Chen, M. Collette, Z. Czaban, S. Erikstad, V. Giuglea, X. Jiang, P. Rigo, F. Roland, Y. Takaoka, V. Zanic, Committee iv.2 (design methods), in: W. Fricke, R. Bronsart (Eds.), 18th International Ship and Offshore Structures Congress, Schiffbautechnische Gesellschaft.

[31] M. Collette, H. K. Jeong, I. Ilnytskiy, I. Lazakis, L. Moro, M. Ventura, M. Toyoda, , M. Sicchiero, P. Georgiev, R. Bronsart, S. O. Erikstad, V. Giuglea, V. Zanic, Y. Chen, Z. Sekulski, Committee iv.2 (design methods), in: C. G. Soares, Y. Garbatov (Eds.), 19th International Ship and Offshore Structures Congress, CRC Press.

[32] A. H. Brodtkorb, U. D. Nielsen, A. J. Sørensen, Sea State Estimation Using Model-scale DP Measurements, in: Proc. of MTS/IEEE OCEANS15, Washington, DC, USA, 2015.

[33] P. A. Brodtkorb, P. Johannesson, G. Lindgren, I. Rychlik, J. Ryden, E. Sjö, M. Sköld, WAFO - a Matlab Toolbox for Analysis of Random Waves and Loads, (The WAFO package can be downloaded freely on the internet.) (August 2000).

[34] J. Dufour, A. Farhat, L. Gardiol, L. Khalaf, Simulation-based Finite Sample Normality Tests in Linear Regression, The Econometrics Journal 1 (1998) 154-173. 
[35] N. Razali, Y. Wah, Power comparisons of ShapiroWilk, KolmogorovSmirnov, Lilliefors and AndersonDarling tests, Journal of Statistical Modeling and Analytics 2 (2011) 21-33.

[36] N. e-Handbook of Statistical Methods, What are statistical tests? (URL date 22-03-2017).

[37] Wikipedia, Null hypothesis (URL date 22-03-2017).

[38] N. e-Handbook of Statistical Methods, Anderson-darling test (URL date 22-03-2017).

[39] Wikipedia, Anderson-darling test (URL date 22-03-2017).

[40] M. Kendall, On the Analysis of Oscillatory Time-Series, Journal of the Royal Statistical Society 108 (1945) 93-141.

[41] T. Hilmer, E. Thornhill, Deterministic wave predictions from the WaMoS II, in: Proc. of MTS/IEEE OCEANS, Taipei, China, 2014.

[42] A. Cameron, F. Windmeijer, An R-squared measure of goodness of fit for some common nonlinear regression models, Jornal of Econometrics 77 (1997) 329-342.

[43] N. Draper, H. Smith, Applied Regression Analysis, 3rd Edition, Wiley, 1998.

[44] Wikipedia, Coefficient of determination (URL date 13-03-2017). 
Appendix A. Prediction and measurement sequences
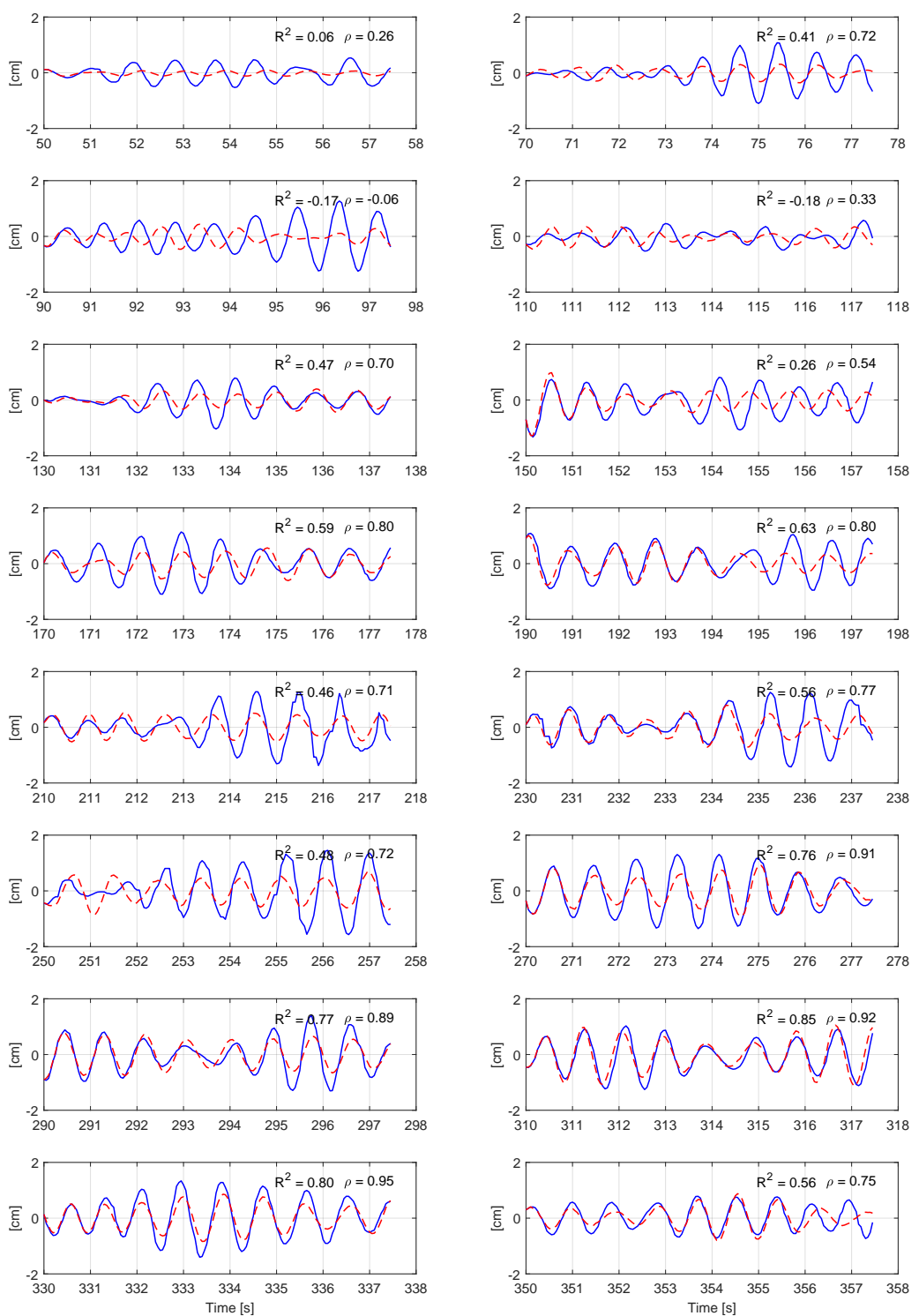

Figure A.13: Heave response sequences of Case 2a; blue full line is measurement and dashed red line is prediction. The determination and correlation coefficients, $R^{2}$ respectively $\rho$, are seen in the upper right-hand corner. 

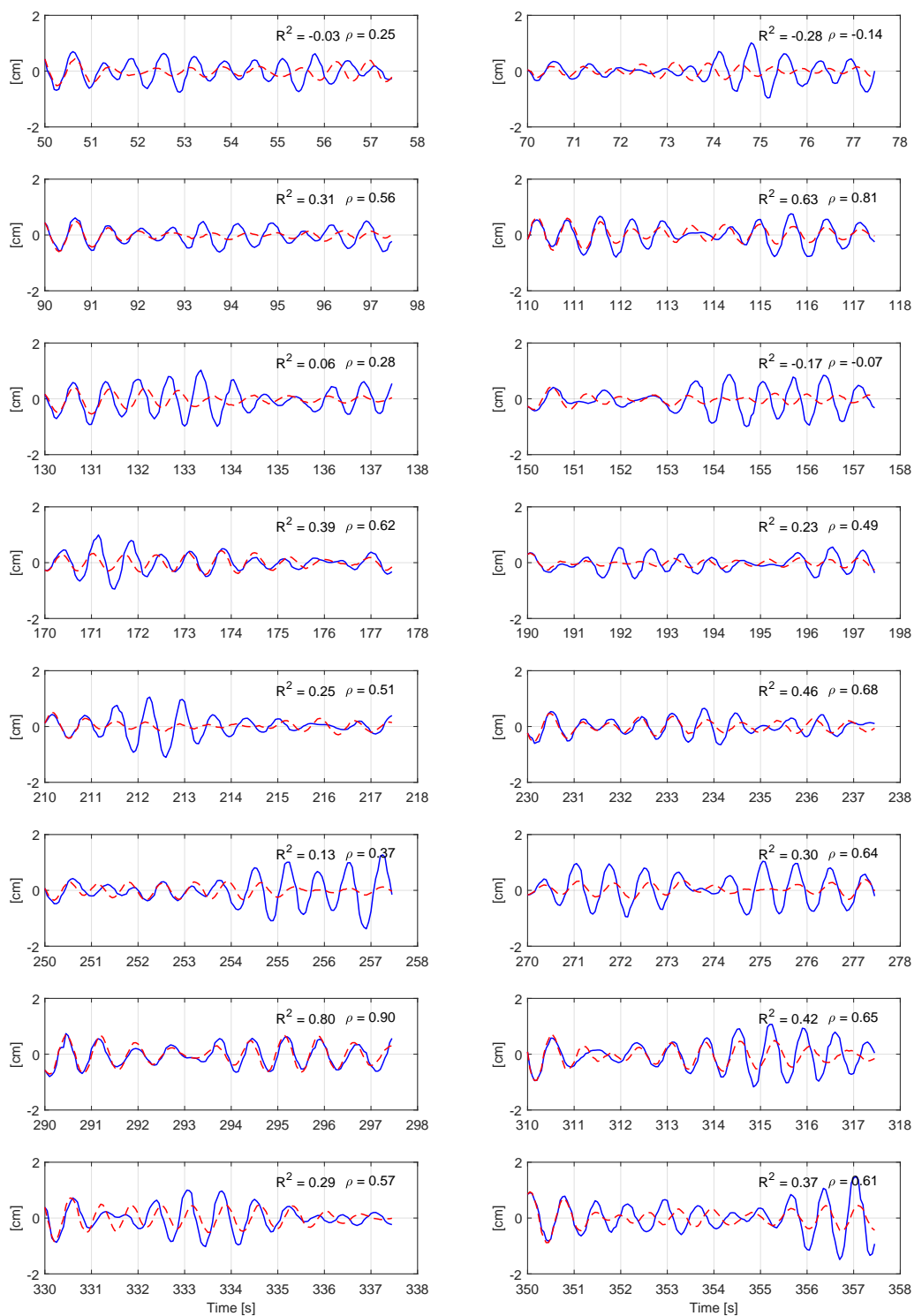

Figure A.14: Heave response sequences of Case 2b; blue full line is measurement and dashed red line is prediction. The determination and correlation coefficients, $R^{2}$ respectively $\rho$, are seen in the upper right-hand corner. 

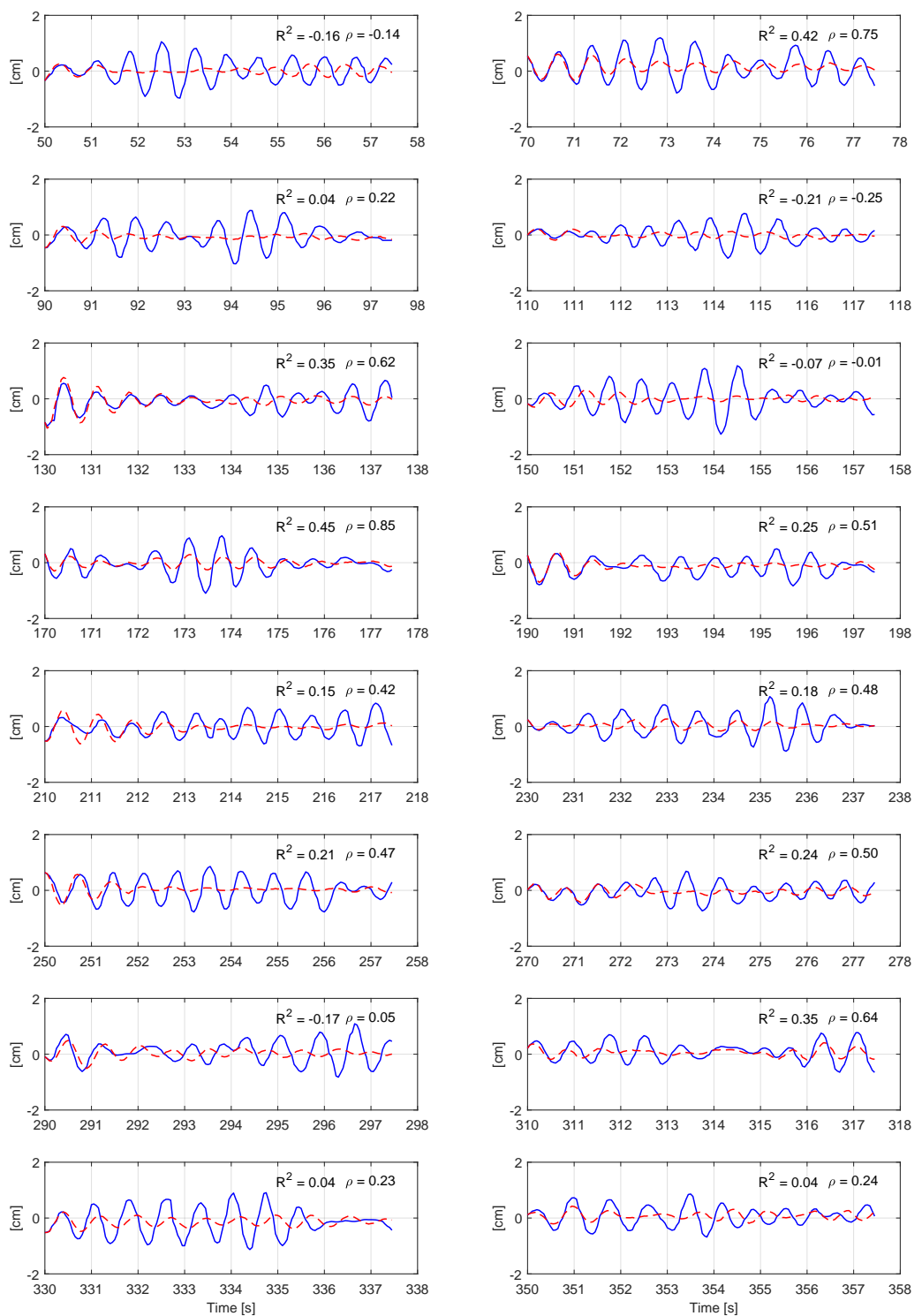

Figure A.15: Heave response sequences of Case 2c; blue full line is measurement and dashed red line is prediction. The determination and correlation coefficients, $R^{2}$ respectively $\rho$, are seen in the upper right-hand corner. 
Appendix B. Full set of data sequences and pair-wise comparisons
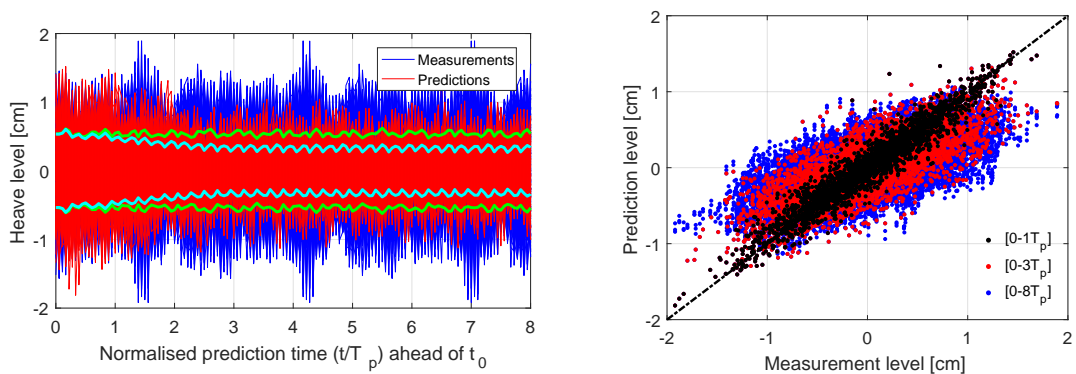

Figure B.16: Case 2a: Heave data sequences (left) and pair-wise comparison (right) of predictions and measurement. The plots are identical to the plots in Figures 7 and 8.
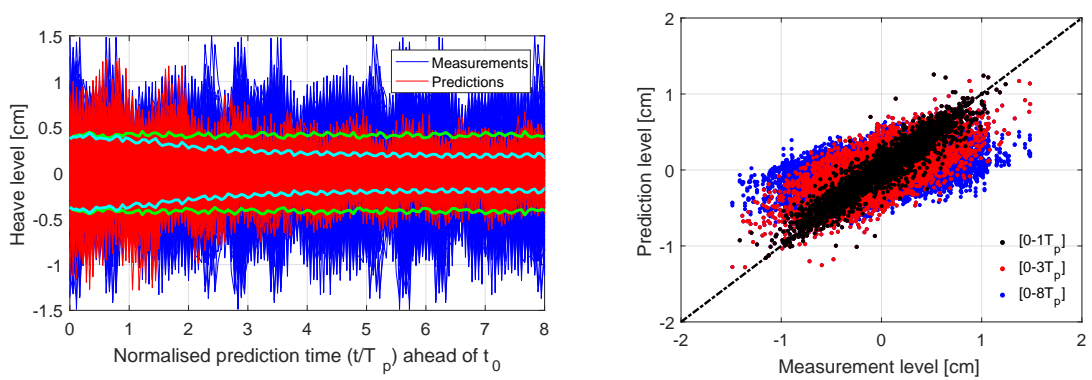

Figure B.17: Case 2b: Heave data sequences (left) and pair-wise comparison (right) of predictions and measurement.
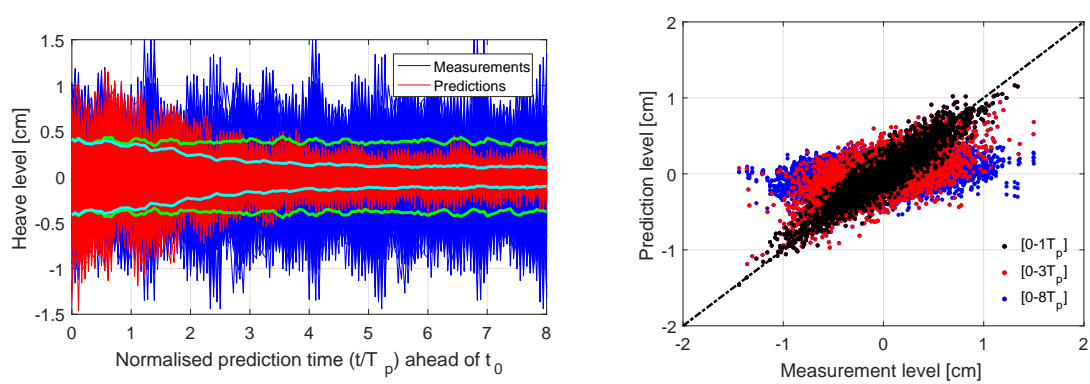

Figure B.18: Case 2c: Heave data sequences (left) and pair-wise comparison (right) of predictions and measurement. 
Appendix C. Determination and correlation coefficients

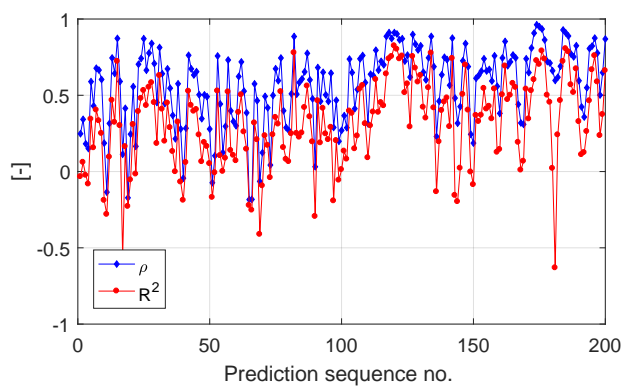

Figure C.19: Determination coefficients $R^{2}$ (red) and correlation coefficients $\rho$ (blue) of Case $2 b$.



Figure C.20: Determination coefficients $R^{2}$ (red) and correlation coefficients $\rho$ (blue) of Case $2 c$. 